\title{
GLOBAL BRANCH OF SOLUTIONS FOR NON-LINEAR SCHRÖDINGER EQUATIONS WITH DEEPENING POTENTIAL WELL
}

\section{A. STUART AND HUAN-SONG ZHOU}

\section{Introduction}

In this paper, we consider the following non-linear Schrödinger equation:

$$
\left\{\begin{array}{l}
-\Delta u+V_{\lambda}(x) u=f(u) \text { in } \mathbb{R}^{N}, \\
u \in H^{1}\left(\mathbb{R}^{N}\right) \text { with } u \neq 0, N \geqslant 1,
\end{array}\right.
$$

where the potential $V_{\lambda}(x)=1+\lambda g(x)$ with $\lambda>0$ and the functions $f$ and $g$ have the following properties:

(F1) $f \in C^{1}(\mathbb{R}, \mathbb{R})$ with $f(s) / s \rightarrow 0$ as $s \rightarrow 0$;

(F2) there exists $\alpha \in(0, \infty)$ such that

$$
f(s) / s \rightarrow \alpha+1 \quad \text { as }|s| \rightarrow+\infty
$$

and

$$
0 \leqslant f(s) / s \leqslant \alpha+1 \quad \text { for all } s \neq 0
$$

(G1) $g \in L^{\infty}\left(\mathbb{R}^{N}, \mathbb{R}\right)$ and there exists a non-empty bounded open set $\Omega \subset \mathbb{R}^{N}$ with Lipschitz boundary such that

$$
g(x) \equiv 0 \text { on } \bar{\Omega}, \quad g(x) \in(0,1] \quad \text { on } \mathbb{R}^{N} \backslash \bar{\Omega} \quad \text { and } \quad \lim _{|x| \rightarrow+\infty} g(x)=1 .
$$

Condition (G1) means that $V_{\lambda}$ represents a potential well whose depth is controlled by the parameter $\lambda$. The non-linear problem (1.1) with a deep potential well ( $\lambda$ large) has been studied by several authors using variational methods under various conditions on $f$. For example, if $f(s)$ is odd and superlinear in $s$ (that is, $\alpha=\infty)$, Bartsch, Pankov and Wang in their papers [2, 3] proved that (1.1) has many solutions in $H^{1}\left(\mathbb{R}^{N}\right)$ for large $\lambda$. If $f(s)$ is odd and asymptotically linear at infinity $(\alpha<\infty)$, van Heerden and Wang in their recent paper [12] proved that if $\alpha>\xi_{1}$, then problem (1.1) still has many solutions for $\lambda$ large, where $\xi_{1}$ is the first eigenvalue of the following Dirichlet problem:

$$
\left\{\begin{array}{l}
-\Delta \varphi=\xi \varphi \text { in } \Omega, \\
\varphi \in H_{0}^{1}(\Omega), \quad \Omega \text { is given by }(\mathrm{G} 1) .
\end{array}\right.
$$

See also $[\mathbf{4}, \mathbf{6}, \mathbf{1 1}]$ for further interesting developments in this direction.

Our work concerns the existence of positive solutions of (1.1) for values of $\lambda$ that are not necessarily large and, in this respect, it is more closely related to some work [14] by Jeanjean and Tanaka. In our notation, they show that (1.1) has at least one

Received 7 January 2005.

2000 Mathematics Subject Classification 35J60, 35B32, 58J55.

The second author was supported by NSFC 10571174, EPFL-IACS and EPFL-CIB. 
positive solution provided that

(i) $\alpha+1>\inf \sigma\left(-\Delta+V_{\lambda}\right)$ and

(ii) there exists $\delta>0$ such that

$$
\lambda+1-\delta>\frac{2}{s^{2}} \int_{0}^{s} f(t) d t \quad \text { for all } s>0,
$$

where $\sigma(S)$ denotes the spectrum of a self-adjoint operator $S$ in $L^{2}\left(\mathbb{R}^{N}\right)$. More recently, Liu and Wang [15] have generalized the result in [14], but in our situation their hypotheses still reduce to (i) and (ii).

The existence results in [14] and [15] are based on concentration-compactness arguments and the fact that the mountain pass geometry occurs for the associated energy functional due to (i) and (ii). In our approach the asymptotic linearization of (1.1) plays a key role and leads to a reformulation of (i) and (ii). On a formal level, this asymptotic linearization is

$$
\begin{aligned}
-\Delta u+V_{\lambda} u & =(\alpha+1) u \text { on } \mathbb{R}^{N}, \quad \text { or equivalently, } \\
-\Delta u-\alpha u+\lambda g u & =0
\end{aligned}
$$

but, as we show in Appendix B, (1.1) is not asymptotically linear in the rigorous sense and we have to resort to a truncation procedure to overcome this technical difficulty. The relevant spectral theory for (1.3) is developed in $[\mathbf{2 3}]$ and it establishes, for $\alpha$ in a suitable range, the existence of a unique eigenvalue $\lambda=\Lambda(\alpha)$ having a positive eigenfunction in $H^{1}\left(\mathbb{R}^{N}\right)$. Alternatively, $\Lambda(\alpha)$ is characterized variationally as the unique value of $\lambda$ for which $\Sigma^{\alpha}(\lambda)=0$ where

$$
\Sigma^{\alpha}(\lambda)=\inf \sigma(-\Delta-\alpha+\lambda g)
$$

and $\sigma(-\Delta-\alpha+\lambda g)$ denotes the spectrum of the Schrödinger operator $-\Delta-\alpha+\lambda g$. The conditions (i) and (ii) of Jeanjean and Tanaka can be reformulated as

$$
\text { (i) }{ }^{\prime} \lambda<\Lambda(\alpha) \text { and (ii) } \alpha<\lambda \text {, respectively, }
$$

and it also follows from our main result that (1.1) has both a positive and a negative solution for each $\lambda$ in the interval $(\alpha, \Lambda(\alpha))$.

However, our goal is to investigate how the positive and negative solutions of (1.1) depend on $\lambda$. We use topological methods to obtain the existence of connected sets $\mathcal{D}^{ \pm}$of positive and negative solutions of (1.1) in $\mathbb{R} \times W^{2, p}\left(\mathbb{R}^{N}\right)$, where $p \in$ $[2, \infty) \cap\left(\frac{1}{2} N, \infty\right)$, that cover the interval $(\alpha, \Lambda(\alpha))$ in the sense that

$$
P \mathcal{D}^{ \pm}=(\alpha, \Lambda(\alpha)) \text { where } P(\lambda, u)=\lambda,
$$

and furthermore,

$$
\lim _{\lambda \rightarrow \Lambda(\alpha)-}\left\|u_{\lambda}\right\|_{L^{\infty}\left(\mathbb{R}^{N}\right)}=\lim _{\lambda \rightarrow \Lambda(\alpha)-}\left\|u_{\lambda}\right\|_{W^{2, p}\left(\mathbb{R}^{N}\right)}=\infty, \quad \text { for }\left(\lambda, u_{\lambda}\right) \in \mathcal{D}^{ \pm} .
$$

This latter property of the branches is sometimes referred to as asymptotic bifurcation (or bifurcation from infinity) as $\lambda$ approaches $\Lambda(\alpha)$. Following Rabinowitz [20] and Toland [24], we use the inversion $v=u /\|u\|^{2}$ to replace (1.1) by

$$
-\Delta v+V_{\lambda} v=\|v\|^{2} f\left(\frac{v}{\|v\|^{2}}\right),
$$

with a view to proving that there is global bifurcation from $(\Lambda(\alpha), 0)$ in $\mathbb{R} \times W^{2, p}\left(\mathbb{R}^{N}\right)$ for this auxiliary problem. However, unlike the analogous situation on a bounded domain in $\mathbb{R}^{N}$, the problem (1.1) is not asymptotically linear in the 
rigorous sense (see Appendix B) and consequently the auxiliary problem (1.4) is not Fréchet differentiable at $v=0$. For this reason, we first replace (1.1) by a truncated problem which is asymptotically linear and for which the standard approach, based on the inversion and the use of a topological degree, is applicable. Due to the lack of compactness that arises from the fact that even the truncated problem is posed on $\mathbb{R}^{N}$, we cannot use the classical Leray-Schauder degree and we appeal to a degree for Fredholm maps. In its most widely used form, this degree is defined for $C^{1}$-Fredholm maps of index 0 that are proper on closed bounded subsets, [9, 16]. However, although inversion of the truncated version of (1.1) does lead to a map that is Fréchet differentiable everywhere, this map cannot be continuously differentiable at $v=0$; see Lemma B.3 in Appendix B. Therefore we have to use a recent extension of the degree to continuous compact perturbations of $C^{1}$-Fredholm maps due to Rabier and Salter [18]. In the usual way, this degree leads to a global result, in the spirit of the original one due to Rabinowitz about bifurcation from the trivial solution $(\Lambda(\alpha), 0)$ of the inverted truncated problem. By a limiting procedure, we obtain a similar conclusion about global bifurcation for the inverted version of (1.1). Finally, to obtain the desired information about a connected set of solutions of the problem (1.1) itself, we use some refinements of the more standard methods involving connectedness that were introduced by Alexander $[\mathbf{1}]$.

The various steps that we have just sketched are presented in the following way.

Section 2: preliminary results and statements of the main theorems concerning the asymptotic linearization (1.3) and the problem (1.1).

Section 3: definition of the truncated problem and proofs of some preparatory lemmas concerning the inversion and the associated auxiliary problems.

Section 4: proof of global bifurcation at $\Lambda(\alpha)$ from the trivial solution for the inverted truncated problem.

Section 5: global bifurcation for the inverted version of (1.1) and deduction of the main conclusions concerning (1.1) from this, including the proof of Theorem 2.3.

Appendix A: a global bifurcation theorem for compact perturbations of $C^{1}$ Fredholm maps of index zero.

Appendix B: on asymptotic linearity.

Appendix C: lemmas about point set topology.

Notation. Throughout this paper, we denote the usual norm of $L^{p}\left(\mathbb{R}^{N}\right)$ for $1 \leqslant p \leqslant+\infty$ by $|\cdot|_{p}$. By (F1) and (F2), we may define two functions $h$ and $k$ in $C(\mathbb{R}, \mathbb{R})$ having the following properties:

$$
\begin{aligned}
& f(s)=h(s) s \\
& \text { with } \quad \lim _{s \rightarrow 0} h(s)=0, \quad \lim _{|s| \rightarrow+\infty} h(s)=\alpha+1 \quad \text { and } \quad 0 \leqslant h(s) \leqslant \alpha+1 . \\
& k(s)=\alpha+1-h(s) \\
& \text { with } \quad \lim _{s \rightarrow 0} k(s)=\alpha+1, \quad \lim _{|s| \rightarrow+\infty} k(s)=0 \quad \text { and } \quad 0 \leqslant k(s) \leqslant \alpha+1 .
\end{aligned}
$$

Moreover, let $\varphi_{1} \in H_{0}^{1}(\Omega)$ denote the unique eigenfunction of (1.2) for the eigenvalue $\xi_{1}$ satisfying the conditions

$$
\int_{\Omega} \varphi_{1}^{2} d x=1 \quad \text { and } \quad \varphi_{1}>0 \quad \text { on } \Omega .
$$


Acknowledgements. The authors express their sincere thanks to Professor P. J. Rabier for his helpful comments. Both authors thank the Bernoulli Centre at EPFL where much of this work was carried out.

\section{The main results}

We begin by introducing a quantity $\Gamma$ that plays a fundamental role in our study of (1.1).

To motivate this definition, observe that if $u$ satisfies (1.1) and $\lambda \geqslant \alpha$, it is easily seen that (F1) and (F2) imply that

$$
\begin{aligned}
(\alpha+1) \int_{\mathbb{R}^{N}} u^{2} d x & >\int_{\mathbb{R}^{N}} f(u) u d x \\
& =\int_{\mathbb{R}^{N}}\left\{|\nabla u|^{2}+(1+\lambda g) u^{2}\right\} d x \\
& \geqslant \int_{\mathbb{R}^{N}}\left\{|\nabla u|^{2}+(1+\alpha g) u^{2}\right\} d x
\end{aligned}
$$

and so

$$
\int_{\mathbb{R}^{N}}|\nabla u|^{2}<\alpha \int_{\mathbb{R}^{N}}(1-g) u^{2} d x
$$

Setting

$$
\Gamma=\inf \left\{\int_{\mathbb{R}^{N}}|\nabla u|^{2} d x: u \in H^{1}\left(\mathbb{R}^{N}\right) \text { and } \int_{\mathbb{R}^{N}}(1-g) u^{2} d x=1\right\},
$$

one finds that $\alpha>\Gamma$ is a necessary condition for (1.1) to have solutions with $\lambda \geqslant \alpha$.

Clearly, $\Gamma \geqslant 0$ and as we show in $[\mathbf{2 3}], \Gamma<\xi_{1}$. In $[\mathbf{2 3}]$ we also establish estimates for $\Gamma$; in particular, we show that $\Gamma=0$ for $N=1$ and 2 . As our main result shows, the conditions (F1), (F2) and (G1) with $\Gamma<\alpha<\xi_{1}$ are sufficient to ensure that the problem (1.1) has positive solutions with $\lambda>\alpha$. The quantity $\Gamma$ does not appear explicitly in [14] and [15], but their hypothesis (ii) implies that $\lambda \geqslant \alpha$ and then we can deduce from (i) that $\alpha>\Gamma$.

To prove the asymptotic bifurcation result, we study first the following formal asymptotic linearization of (1.1) at infinity (see (1.3)):

$$
\left\{\begin{array}{l}
-\Delta u-\alpha u+\lambda g u=0 \text { in } \mathbb{R}^{N} \\
u \in H^{1}\left(\mathbb{R}^{N}\right), \quad \lambda>0 .
\end{array}\right.
$$

A number $\lambda>0$ is said to be an eigenvalue of (2.2) whenever there exists $u \in$ $H^{1}\left(\mathbb{R}^{N}\right) \backslash\{0\}$ such that

$$
\int_{\mathbb{R}^{N}}[\nabla u \cdot \nabla v-\alpha u v+\lambda g u v] d x=0 \quad \text { for all } v \in H^{1}\left(\mathbb{R}^{N}\right) .
$$

For the discussion of the non-linear problem (1.1), it is desirable to take advantage of the additional regularity of solutions that follows from our assumptions.

Proposition 2.1. (1) Let the conditions (F1), (F2) and (G1) be satisfied and let $u \in H^{1}\left(\mathbb{R}^{N}\right)$ satisfy (1.1). Then $u \in W^{2, p}\left(\mathbb{R}^{N}\right)$ for all $p \in[2, \infty)$ and hence $u \in C^{1}\left(\mathbb{R}^{N}\right)$ with $\lim _{|x| \rightarrow \infty} u(x)=0$ and $\lim _{|x| \rightarrow \infty} \nabla u(x)=0$.

(2) If $g$ satisfies (G1) and $v \in H^{1}\left(\mathbb{R}^{N}\right)$ is an eigenfunction of (2.2), then $v \in$ $W^{2, p}\left(\mathbb{R}^{N}\right)$ for all $p \in[2, \infty)$. 
Proof. Setting $H(x)=f(u(x)) / u(x)$ if $u(x) \neq 0$ and $H(x)=0$ if $u(x)=0$, then, if $u$ satisfies (1.1), we have

$$
\int_{\mathbb{R}^{N}}(\nabla u \cdot \nabla z+w u z) d x=0 \quad \text { for all } z \in C_{0}^{\infty}\left(\mathbb{R}^{N}\right),
$$

where $w \equiv 1+\lambda g-H \in L^{\infty}\left(\mathbb{R}^{N}\right)$. The conclusions (1) and (2) now follow by standard elliptic regularity and boot-strapping. See [22, Corollary 2.15] for example, or $[\mathbf{1 7}]$ for a deeper discussion.

The main results of our paper [23] concerning the linearized problem (2.2) can be summarized as follows.

Proposition 2.2. Let the condition (G1) be satisfied.

(i) If $\alpha \geqslant \xi_{1}$, then there is no eigenvalue of $(2.2)$ in $[\alpha, \infty)$ with a non-negative eigenfunction.

(ii) If $\Gamma<\alpha<\xi_{1}$, then there exists a unique eigenvalue $\lambda=\Lambda(\alpha)$ of (2.2) having a positive eigenfunction. Furthermore, $\Lambda(\alpha)>\alpha$, and it is simple in the sense that $\operatorname{ker}(-\Delta-\alpha+\Lambda(\alpha) g)=\operatorname{span}\left\{u_{\Lambda(\alpha)}\right\}$ where $u_{\Lambda(\alpha)}>0$ on $\mathbb{R}^{N}$. All other eigenvalues of (2.2) are less than $\Lambda(\alpha)$ and their eigenfunctions change sign.

(iii) The function $\Lambda \in C^{\infty}\left(\left(\Gamma, \xi_{1}\right)\right)$ and is strictly increasing with

$$
\lim _{\alpha \rightarrow \Gamma+} \Lambda(\alpha)=\Gamma \quad \text { and } \quad \lim _{\alpha \rightarrow \xi_{1}-} \Lambda(\alpha)=+\infty .
$$

(iv) For $\Gamma<\alpha<\xi_{1}, \Lambda(\alpha)$ is characterized as the unique value of $\lambda$ for which $\Sigma^{\alpha}(\lambda)=0$ where

$$
\Sigma^{\alpha}(\lambda)=\inf \left\{a_{\lambda}(u): u \in H^{1}\left(\mathbb{R}^{N}\right) \text { and } \int_{\mathbb{R}^{N}} u^{2} d x=1\right\}
$$

and

$$
a_{\lambda}(u)=\int_{\mathbb{R}^{N}}\left(|\nabla u|^{2}-\alpha u^{2}+\lambda g u^{2}\right) d x .
$$

In other words, $\Lambda(\alpha)$ is the unique value of $\lambda$ for which 0 is the infimum of the spectrum of the Schrödinger operator

$$
A_{\lambda}^{\alpha} u=-\Delta u-(\alpha-\lambda g) u .
$$

(v) If $\alpha \leqslant \Gamma$, the problem (2.2) has no eigenvalues $\lambda$ in the interval $(\alpha, \infty)$.

We can now state our main result concerning the non-linear problem (1.1).

TheOREm 2.3. Let the conditions (F1), (F2) and (G1) hold with $\Gamma<\alpha<\xi_{1}$ and fix $p \in\left(\frac{1}{2} N, \infty\right) \cap[2,+\infty)$. Then there exist two connected subsets $\mathcal{D}^{+}$and $\mathcal{D}^{-}$ of $\mathbb{R} \times W^{2, p}\left(\mathbb{R}^{N}\right)$, whose elements $(\lambda, u)$ are, respectively, positive and negative solutions of problem (1.1), such that

$$
\inf \left\{\lambda:(\lambda, u) \in \mathcal{D}^{ \pm}\right\}=\alpha \quad \text { and } \sup \left\{\lambda:(\lambda, u) \in \mathcal{D}^{ \pm}\right\}=\Lambda(\alpha),
$$

where $\Lambda(\alpha)$ is given by Proposition 2.2(ii). Furthermore, $\mathcal{D}^{ \pm}$is bounded away from the line of trivial solutions $\mathbb{R} \times\{0\}$ and if $\left\{\left(\lambda_{n}, u_{n}\right)\right\} \subset \mathcal{D}^{ \pm}$with $\lambda_{n} \stackrel{n}{\rightarrow} \lambda>\alpha$, then $\max _{x \in \mathbb{R}^{N}}\left|u_{n}(x)\right| \stackrel{n}{\rightarrow} \infty$ if and only if $\lambda=\Lambda(\alpha)$. 


\section{The truncated problem and some estimates}

Throughout this section we suppose that the conditions (F1), (F2) and (G1) are satisfied with $\Gamma<\alpha<\xi_{1}$. Furthermore, $p \in\left(\frac{1}{2} N, \infty\right) \cap[2,+\infty)$ is fixed and we set

$$
\begin{aligned}
& X=W^{2, p}\left(\mathbb{R}^{N}\right) \quad \text { with }\|\cdot\|=\|\cdot\|_{W^{2, p}\left(\mathbb{R}^{N}\right),}, \\
& Y=L^{p}\left(\mathbb{R}^{N}\right) \\
& \text { with }|\cdot|_{p}=\|\cdot\|_{L^{p}\left(\mathbb{R}^{N}\right) .}
\end{aligned}
$$

In the notation (1.6), the equation (1.1) is equivalent to

$$
-\Delta u-\alpha u+\lambda g u+k(u) u=0
$$

where $k(s) \rightarrow \alpha+1$ as $s \rightarrow 0$ and $k(s) \rightarrow 0$ as $|s| \rightarrow \infty$. This means that, at least formally, $-\Delta u-\alpha u+\lambda g u$ is the asymptotic linearization of $-\Delta u-\alpha u+\lambda g u+k(u) u$. However, since we are considering (1.1) on $\mathbb{R}^{N}$, it can be shown that (see Lemma B.1)

$$
|k(u) u|_{p} /\|u\| \nrightarrow 0 \quad \text { as }\|u\| \rightarrow \infty .
$$

This leads us to introduce the following truncated problem. For $n \in \mathbb{N} \backslash\{0\}$, let

$$
\psi_{n}(x)= \begin{cases}1 & \text { if }|x| \leqslant n \\ 0 & \text { if }|x|>n .\end{cases}
$$

The following result is an immediate consequence of Lemma B.2.

Lemma 3.1. Let $n \in \mathbb{N} \backslash\{0\}$ be fixed. The mapping $Q_{n}: X \rightarrow Y$ defined by $Q_{n}(u)=\psi_{n} k(u) u$ for $u \in X$ is continuous, bounded, compact and asymptotically linear with asymptotic derivative equal to zero.

Define $L(\lambda): W^{2, p}\left(\mathbb{R}^{N}\right) \rightarrow L^{p}\left(\mathbb{R}^{N}\right)$ and $G: \mathbb{R} \times W^{2, p}\left(\mathbb{R}^{N}\right) \rightarrow L^{p}\left(\mathbb{R}^{N}\right)$ by

$$
L(\lambda) u=-\Delta u-\alpha u+\lambda g(x) u
$$

and

$$
G(\lambda, u)=L(\lambda) u+k(u) u
$$

Let

$$
G_{n}(\lambda, u)=L(\lambda) u+\psi_{n} k(u) u .
$$

In order to exploit the asymptotic linearity of $G_{n}$ that follows from Lemma 3.1, we introduce

$$
F_{n}(\lambda, v)= \begin{cases}\|v\|^{2} G_{n}\left(\lambda, v /\|v\|^{2}\right)=L(\lambda) v+\psi_{n} k\left(v /\|v\|^{2}\right) v & \text { if } v \neq 0 \\ 0 & \text { if } v=0\end{cases}
$$

Similarly, we set

$$
F(\lambda, v)= \begin{cases}L(\lambda) v+k\left(v /\|v\|^{2}\right) v & \text { if } v \neq 0 \\ 0 & \text { if } v=0\end{cases}
$$

REMARK 3.1. It follows from Proposition 2.1 that any solution $u \in H^{1}\left(\mathbb{R}^{N}\right)$ of (1.1) belongs to $X$ and $G(\lambda, u)=0$. Conversely, if $(\lambda, u) \in \mathbb{R} \times X$ with $\lambda>\alpha$ and $G(\lambda, u)=0$, it follows from Lemma 3.4 below that $u \in L^{2}\left(\mathbb{R}^{N}\right)$. Since $0 \leqslant$ $k(s) \leqslant(\alpha+1)$ for all $s \in \mathbb{R}$, it follows that $u \in H^{1}\left(\mathbb{R}^{N}\right)$; see [22, Theorem 2.8], for 
example. Hence, at least for $\lambda>\alpha$, the problem (1.1) is equivalent to the equation $G(\lambda, u)=0$ for $u \in X$.

Using the inversion, $u \mapsto v=u /\|u\|^{2}$, one transforms non-trivial solutions of $G(\lambda, u)=0$ and $G_{n}(\lambda, u)=0$ to non-trivial solutions of $F(\lambda, v)=0$ and $F_{n}(\lambda, v)=$ 0 , respectively, and vice versa. This leads us to introduce the following sets:

$$
\begin{aligned}
\mathcal{Z}_{n} & =\left\{(\lambda, v) \in(\alpha, \infty) \times X: F_{n}(\lambda, v)=0 \text { and } v \not \equiv 0\right\}, \\
\mathcal{Z} & =\{(\lambda, v) \in(\alpha, \infty) \times X: F(\lambda, v)=0 \text { and } v \not \equiv 0\} .
\end{aligned}
$$

Next, we set

$$
K_{n}(v)= \begin{cases}\psi_{n} k\left(v /\|v\|^{2}\right) v & \text { for } v \in X \backslash\{0\} \\ 0 & \text { for } v \equiv 0\end{cases}
$$

where $\psi_{n}$ is defined by (3.1), so that the equation $F_{n}(\lambda, v)=0$ becomes

$$
L(\lambda) v+K_{n}(v)=0 .
$$

Since $0 \leqslant k(s) \leqslant \alpha+1$ for all $s \in \mathbb{R}$, it is clear that $K_{n}(v) \in Y$ for all $v \in X$.

Lemma 3.2. For all $n \in \mathbb{N}, K_{n} \in C(X, Y) \cap C^{1}(X \backslash\{0\}, Y), K_{n}: X \rightarrow Y$ is compact and it is also Fréchet differentiable at 0 with $K_{n}^{\prime}(0)=0$.

Proof. Apart from the assertion about compactness, all these conclusions follow from Lemma B.3.

For the compactness, let us consider a bounded sequence $\left\{v_{i}\right\}$ in $X$. Passing to a subsequence we may assume that

$$
\begin{aligned}
& v_{i} \stackrel{i}{\rightarrow} v \text { weakly in } X, \\
& v_{i} \stackrel{i}{\rightarrow} v \text { uniformly on } B_{n}=\{x \in \mathbb{N}:|x|<n\}, \\
& \left\|v_{i}\right\| \stackrel{i}{\rightarrow} c \geqslant 0 .
\end{aligned}
$$

Since $\left|K_{n}\left(v_{i}\right)\right|_{p} \leqslant(\alpha+1)\left|v_{i}\right|_{p} \leqslant(\alpha+1)\left\|v_{i}\right\|$, it follows that $\left|K_{n}\left(v_{i}\right)\right|_{p} \stackrel{i}{\rightarrow} 0$ if $c=0$. We suppose henceforth that $c>0$. Now

$$
v_{i} /\left\|v_{i}\right\|^{2} \stackrel{i}{\rightarrow} v / c^{2} \quad \text { uniformly on } B_{n}
$$

and so

$$
k\left(\frac{v_{i}}{\left\|v_{i}\right\|^{2}}\right) v_{i} \stackrel{i}{\rightarrow} k\left(\frac{v}{c^{2}}\right) v \quad \text { and } \quad \psi_{n} k\left(\frac{v_{i}}{\left\|v_{i}\right\|^{2}}\right) v_{i} \stackrel{i}{\rightarrow} \psi_{n} k\left(\frac{v}{c^{2}}\right) v \quad \text { uniformly on } B_{n},
$$

showing that $K_{n}\left(v_{i}\right)$ converges to $\psi_{n} k\left(v / c^{2}\right) v$ in $Y$. This proves the compactness of $K_{n}: X \rightarrow Y$.

We end this section with some results concerning a priori bounds and exponential decay.

Lemma 3.3. There exists $T>0$ such that:

(i) $\|v\| \leqslant T$ for all $(\lambda, v) \in \mathcal{Z}$;

(ii) for any given $\mu>\alpha$, there exists $N_{\mu} \in \mathbb{N}$ such that $\|v\| \leqslant T$ for all $(\lambda, v) \in \mathcal{Z}_{n}$ with $\lambda \geqslant \mu$ and $n \geqslant N_{\mu}$. 
REMARK 3.2. Recalling that $X$ is continuously embedded in $L^{\infty}\left(\mathbb{R}^{N}\right)$, we see that these results imply $L^{\infty}$-bounds too.

Proof of Lemma 3.3. We begin by defining the bound $T$. Since $k(0)=\alpha+1$, there exists a constant $S>0$ such that $k(s) \geqslant \alpha$ for all $|s| \leqslant S$. Also there is a constant $C>0$ such that $|u|_{\infty} \leqslant C\|u\|$ for all $u \in X$. Set $T=C / S$.

(i) Suppose that $v \in X$ with $\|v\| \geqslant T$. Then, for all $x \in \mathbb{R}^{N}$, we have

$$
\frac{|v(x)|}{\|v\|^{2}} \leqslant \frac{|v|_{\infty}}{\|v\|^{2}} \leqslant \frac{C}{\|v\|} \leqslant \frac{C}{T}=S
$$

and so $k\left(v(x) /\|v\|^{2}\right) \geqslant \alpha$. Hence,

$$
-\alpha+\lambda g(x)+k\left(\frac{v(x)}{\|v\|^{2}}\right) \geqslant 0 \quad \text { for all } x \in \mathbb{R}^{N}
$$

since $\lambda g \geqslant 0$, and if $(\lambda, v) \in \mathcal{Z}$, we find that

$$
v(x) \Delta v(x)=v(x)^{2}\left[-\alpha+\lambda g(x)+k\left(\frac{v(x)}{\|v\|^{2}}\right)\right] \geqslant 0 \quad \text { for all } x \in \mathbb{R}^{N} .
$$

The maximum principle now leads to a contradiction as follows. For $\varepsilon>0$, let

$$
D(\varepsilon)=\left\{x \in \mathbb{R}^{N}: v(x)>\varepsilon\right\} .
$$

Recalling that $v \in X \subset C\left(\mathbb{R}^{N}\right)$ and $\lim _{|x| \rightarrow \infty} v(x)=0$, we see that $D(\varepsilon)$ is a bounded open subset of $\mathbb{R}^{N}$ and that $v(x)=\varepsilon$ on $\partial D(\varepsilon)$. But $\Delta v \geqslant 0$ on $D(\varepsilon)$ and so the weak maximum principle (see [10, Theorem 8.1] for example) implies that $v \leqslant \varepsilon$ on $D(\varepsilon)$, a contradiction, unless $D(\varepsilon)=\emptyset$. Hence we must have $v \leqslant 0$ on $\mathbb{R}^{N}$.

But a similar argument shows that we must have $v \geqslant 0$ on $\mathbb{R}^{N}$ too, so that in fact $v \equiv 0$, contradicting the assumptions that $(\lambda, v) \in \mathcal{Z}$ with $\|v\| \geqslant T$.

(ii) Consider $(\lambda, v) \in \mathbb{R} \times X$ with $\lambda \geqslant \mu>\alpha$. There exists $\delta_{\mu}>0$ such that $\mu\left(1-\delta_{\mu}\right)>\alpha$ and, since $\lim _{|x| \rightarrow \infty} g(x)=1$, there exists an integer $N_{\mu}$ such that

$$
g(x) \geqslant 1-\delta_{\mu} \text { for all }|x| \geqslant N_{\mu} .
$$

Hence,

$$
\lambda g(x) \geqslant \mu\left(1-\delta_{\mu}\right)>\alpha \text { for all }|x| \geqslant N_{\mu} .
$$

On the other hand, for $n \geqslant N_{\mu}$ and $|x| \leqslant N_{\mu}$, we have

$$
\psi_{n}(x) k\left(\frac{v(x)}{\|v\|^{2}}\right)=k\left(\frac{v(x)}{\|v\|^{2}}\right) \geqslant \alpha
$$

as in case (i) if $\|v\| \geqslant T$.

Combining (3.11) and (3.12), we find that we again have

$$
v(x) \Delta v(x)=v(x)^{2}\left[-\alpha+\lambda g(x)+\psi_{n}(x) k\left(\frac{v(x)}{\|v\|^{2}}\right)\right] \geqslant 0 \quad \text { for all } x \in \mathbb{R}^{N},
$$

provided that $n \geqslant N_{\mu}$ and the proof can now be completed as in case (i).

The next result establishes some uniform exponential decay of solutions and, in particular, it enables us to derive $L^{p}$-bounds from $L^{\infty}$-bounds. 
Lemma 3.4. Fix $\mu>\alpha$. Then, for any $\varepsilon \in(0,(\mu-\alpha) / \mu)$, there exists $C_{\varepsilon}>0$ such that

$$
|u(x)| \leqslant|u|_{\infty} e^{-\sqrt{\xi}\left(|x|-C_{\varepsilon}\right)} \quad \text { for all } x \in \mathbb{R}^{N},
$$

where $\xi=\mu(1-\varepsilon)-\alpha>0$ for all $(\lambda, u) \in[\mu,+\infty) \times X$ with $u L(\lambda) u \leqslant 0$ on $\mathbb{R}^{N}$.

Proof. Since $\mu>\alpha$, there exists $\varepsilon_{0} \in(0,1)$ such that $\mu\left(1-\varepsilon_{0}\right)>\alpha$ and, since $g(x) \rightarrow 1$ as $|x| \rightarrow \infty$, for any $\varepsilon \in\left(0, \varepsilon_{0}\right)$ there exists $C_{\varepsilon}>0$ such that

$$
g(x) \geqslant 1-\varepsilon \quad \text { for all }|x| \geqslant C_{\varepsilon} \text {. }
$$

Setting $q(x)=|u|_{\infty} e^{-\sqrt{\xi}\left(|x|-C_{\varepsilon}\right)}-u(x)$, we consider the set

$$
D_{\varepsilon}=\left\{x \in \mathbb{R}^{N}:|x| \geqslant C_{\varepsilon} \text { and } q(x)<0\right\} .
$$

Clearly $u(x)>0$ for $x \in D_{\varepsilon}$ and so

$$
\begin{aligned}
0 \geqslant L(\lambda) u=-\Delta u-\alpha u+\lambda g u & \geqslant-\Delta u-\alpha u+\mu(1-\varepsilon) u \\
& =-\Delta u+\xi u, \quad \text { for all } x \in D_{\varepsilon},
\end{aligned}
$$

since $\lambda \geqslant \mu$. Hence, for $x \in D_{\varepsilon}$,

$$
\begin{aligned}
\Delta q(x) & =|u|_{\infty} e^{\sqrt{\xi} C_{\varepsilon}}\left(\xi-\frac{N-1}{|x|} \sqrt{\xi}\right) e^{-\sqrt{\xi}|x|}-\Delta u \\
& \leqslant|u|_{\infty} e^{\sqrt{\xi} C_{\varepsilon}} \xi e^{-\sqrt{\xi}|x|}-\xi u=\xi q(x)<0 .
\end{aligned}
$$

But $q(x) \geqslant 0$ when $|x|=C_{\varepsilon}$ so that $q(x) \geqslant 0$ for all $x \in \partial D_{\varepsilon}$ and $q(x) \rightarrow 0$ as $|x| \rightarrow \infty$. If $D_{\varepsilon} \neq \emptyset$, the weak maximum principle [10, Theorem 8.1] now implies that $q \geqslant 0$ in $D_{\varepsilon}$, a contradiction. Thus we see that $D_{\varepsilon}=\emptyset$ and consequently,

$$
u(x) \leqslant|u|_{\infty} e^{-\sqrt{\xi}\left(|x|-C_{\varepsilon}\right)} \text { for all }|x| \geqslant C_{\varepsilon} .
$$

Replacing $u$ by $-u$, we obtain the desired inequality for $|u(x)|$ in the region $|x| \geqslant C_{\varepsilon}$. But for $|x| \leqslant C_{\varepsilon}$ the estimate is trivial so the proof is complete.

The next result enables us to exploit the properness on closed bounded sets of linear Fredholm operators. In what follows we use some of the notation introduced in Appendix A.

Lemma 3.5. Consider $\lambda>\alpha$ and any $p \in[2, \infty)$.

(i) The map $L(\lambda): X=W^{2, p}\left(\mathbb{R}^{N}\right) \rightarrow L^{p}\left(\mathbb{R}^{N}\right)$ with $p \in[2, \infty)$ is a Fredholm operator of index zero.

(ii) Let $\left\{v_{n}\right\} \subset X, v_{n} \stackrel{n}{\rightarrow} v$ weakly in $X$ and let $\left\{L(\lambda) v_{n}\right\}$ converge strongly in $L^{p}\left(\mathbb{R}^{N}\right)$. Then $v_{n} \stackrel{n}{\rightarrow} v$ strongly in $X$.

Proof. (i) Since $\lim _{|x| \rightarrow \infty}(-\alpha+\lambda g(x))=\lambda-\alpha>0$, this follows from $[\mathbf{1 3}$, Theorem 4.3].

(ii) Since $L(\lambda): X \rightarrow L^{p}\left(\mathbb{R}^{N}\right)$ is a Fredholm operator of index 0 , by $[\mathbf{5}$, Chapter I, Theorem 3.15], there exists $T \in \mathcal{B}\left(L^{p}\left(\mathbb{R}^{N}\right), X\right)$ such that

$$
T L(\lambda)=I+K
$$

where $K: X \rightarrow X$ is a compact linear operator. Let $L(\lambda) v_{n} \stackrel{n}{\rightarrow} w$ strongly in $L^{p}\left(\mathbb{R}^{N}\right)$ for some $w \in L^{p}\left(\mathbb{R}^{N}\right)$; then $T L(\lambda) v_{n} \stackrel{n}{\rightarrow} T w$ strongly in $X$. It follows from 
$T L(\lambda)=I+K$ and since $v_{n} \stackrel{n}{\longrightarrow} v$ weakly in $X$ that

$$
(I+K) v_{n} \stackrel{n}{\rightarrow} T w \text { strongly in } X \quad \text { and } \quad K v_{n} \stackrel{n}{\rightarrow} K v \text { strongly in } X \text {. }
$$

This implies that $v_{n} \stackrel{n}{\rightarrow} T w-K v$ strongly in $X$, and hence that

$$
v_{n} \stackrel{n}{\rightarrow} v=T w-K v \text { strongly in } X \text {. }
$$

We end this section with some results concerning the way in which solutions can approach the line $\mathbb{R} \times\{0\}$ of trivial solutions.

LEMMA 3.6. Let $\left\{\left(\lambda_{n}, v_{n}\right)\right\} \subset \mathbb{R} \times X$ be a sequence such that $\lambda_{n} \stackrel{n}{\rightarrow} \lambda>\alpha$ and $\left\|v_{n}\right\| \stackrel{n}{\rightarrow} 0$ with $\left\|v_{n}\right\| \neq 0$ and $v_{n} L\left(\lambda_{n}\right) v_{n} \leqslant 0$ on $\mathbb{R}^{N}$ for all $n \in \mathbb{N}$. Then:

(i) $v_{n} \in \bigcap_{1 \leqslant r<\infty} L^{r}\left(\mathbb{R}^{N}\right)$ and $\left|k\left(v_{n} /\left\|v_{n}\right\|^{2}\right) v_{n}\right|_{r} /\left\|v_{n}\right\| \stackrel{n}{\rightarrow} 0$ for all $r \in(1,+\infty)$, and

(ii) $\lambda=\Lambda(\alpha)$ if $\left|L\left(\lambda_{n}\right) v_{n}\right|_{p} /\left\|v_{n}\right\| \stackrel{n}{\rightarrow} 0$ and $v_{n}^{2}>0$ on $\mathbb{R}^{N}$ for all $n \in \mathbb{N}$, where $\Lambda(\alpha)>0$ is defined in Proposition 2.2(ii).

Proof. (i) Since $v_{n} L\left(\lambda_{n}\right) v_{n} \leqslant 0$, it follows from Lemma 3.4 that there exist $D>0$ and $\gamma>0$ which are independent of $n$ such that

$$
\left|v_{n}(x)\right| \leqslant D\left|v_{n}\right|_{\infty} e^{-\gamma|x|} \text { for all } x \in \mathbb{R}^{N} .
$$

Using this estimate, one can easily see that $v_{n} \in \bigcap_{1 \leqslant r<\infty} L^{r}\left(\mathbb{R}^{N}\right)$.

We claim that, for every fixed $x \in \mathbb{R}^{N}$,

$$
\left|k\left(\frac{v_{n}(x)}{\left\|v_{n}\right\|^{2}}\right) v_{n}(x)\right| /\left\|v_{n}\right\| \stackrel{n}{\rightarrow} 0 .
$$

By contradiction, suppose that for some $x_{0} \in \mathbb{R}^{N}$ there exist $\delta>0$ and a subsequence $\left\{v_{n_{j}}\right\}$ of $\left\{v_{n}\right\}$ such that

$$
\left|k\left(\frac{v_{n_{j}}\left(x_{0}\right)}{\left\|v_{n_{j}}\right\|^{2}}\right) v_{n_{j}}\left(x_{0}\right)\right| /\left\|v_{n_{j}}\right\| \geqslant \delta \quad \text { for all } n_{j} \in \mathbb{N} .
$$

Let $w_{n_{j}}(x)=v_{n_{j}}(x) /\left\|v_{n_{j}}\right\|$. Then

$$
\left\|w_{n_{j}}\right\|=1 \quad \text { and } \quad\left|w_{n_{j}}\left(x_{0}\right)\right| \leqslant\left|w_{n_{j}}\right|_{\infty} \leqslant C\left\|w_{n_{j}}\right\|=C \text {, }
$$

so we may assume that $w_{n_{j}}\left(x_{0}\right) \stackrel{j}{\rightarrow} \tau$. If $\tau=0$, it is easy to see that

$$
\left|k\left(\frac{v_{n_{j}}\left(x_{0}\right)}{\left\|v_{n_{j}}\right\|^{2}}\right) v_{n_{j}}\left(x_{0}\right)\right| /\left\|v_{n_{j}}\right\|=\left|k\left(\frac{v_{n_{j}}\left(x_{0}\right)}{\left\|v_{n_{j}}\right\|^{2}}\right)\right|\left|w_{n_{j}}\left(x_{0}\right)\right| \stackrel{j}{\rightarrow} 0
$$

since $|k(s)| \leqslant \alpha+1$, contradicting (3.16). If $\tau \neq 0$, then

$$
\frac{v_{n_{j}}\left(x_{0}\right)}{\left\|v_{n_{j}}\right\|^{2}}=\frac{w_{n_{j}}\left(x_{0}\right)}{\left\|v_{n_{j}}\right\|} \stackrel{j}{\rightarrow} \infty
$$

since $\left\|v_{n_{j}}\right\| \stackrel{j}{\rightarrow} 0$. Hence $k\left(v_{n_{j}}\left(x_{0}\right) /\left\|v_{n_{j}}\right\|^{2}\right) \rightarrow 0$, so we still have (3.17), again contradicting (3.16). This proves (3.15).

Since $v_{n} L\left(\lambda_{n}\right) v_{n} \leqslant 0$, it follows from (3.14) that

$$
\left|k\left(\frac{v_{n}(x)}{\left\|v_{n}\right\|^{2}}\right) v_{n}(x)\right| /\left\|v_{n}\right\| \leqslant(\alpha+1) C D e^{-\gamma|x|} \quad \text { for all } x \in \mathbb{R}^{N} .
$$

Therefore, part (i) follows from (3.15) and dominated convergence. 
(ii) Let $w_{n}=v_{n} /\left\|v_{n}\right\|$. Then $\left\|w_{n}\right\|=1$. Since $v_{n}^{2}(x)>0$, on passing to a subsequence, we may assume that either $w_{n}(x)>0$ or $w_{n}(x)<0$ for all $n \in \mathbb{N}$ and, for some $w \in X$,

$$
w_{n} \stackrel{n}{\rightarrow} w \text { weakly in } X \quad \text { with } w \geqslant 0 \text { or } w \leqslant 0 .
$$

By the definition of $L(\lambda)$ (see (3.2)), it is easy to see that

$$
L(\lambda) w_{n}=L\left(\lambda_{n}\right) w_{n}-\left(\lambda_{n}-\lambda\right) g(x) w_{n} \stackrel{n}{\rightarrow} 0 \text { in } Y .
$$

Since $\lambda>\alpha$, by Lemma 3.5(i), we know that $L(\lambda): X \rightarrow Y$ is a Fredholm operator of index 0 . Then it follows from Lemma 3.5 (ii) that $w_{n} \stackrel{n}{\rightarrow} w$ strongly in $X$, and $w \not \equiv 0$ since $\left\|w_{n}\right\|=1$. So $L(\lambda) w=0$ and, as in Remark 3.1, this implies that $w \in H^{1}\left(\mathbb{R}^{\mathbb{N}}\right)$. Therefore, $\lambda=\Lambda(\alpha)$ by Proposition 2.2(ii) since either $w \geqslant 0$ or $w \leqslant 0$.

LEMma 3.7. Let $\left\{\left(\lambda_{m}, v_{m}\right)\right\} \subset \mathcal{Z}_{n}$ with $v_{m}^{2}>0$ on $\mathbb{R}^{N}$, and let $\left\|v_{m}\right\| \stackrel{m}{\rightarrow} 0$ and $\lambda_{m} \stackrel{m}{\rightarrow} \lambda>\alpha$. Then $\lambda=\Lambda(\alpha)$, where $\Lambda(\alpha)>0$ is defined in Proposition 2.2(ii).

Proof. Since $\left(\lambda_{m}, v_{m}\right) \in \mathcal{Z}_{n}$, we have

$$
L\left(\lambda_{m}\right) v_{m}+\psi_{n} k\left(\frac{v_{m}}{\left\|v_{m}\right\|^{2}}\right) v_{m}=0
$$

Setting $w_{m}(x)=v_{m}(x) /\left\|v_{m}\right\|^{2}$, we see that this becomes

$$
L\left(\lambda_{m}\right) w_{m}+\psi_{n} k\left(w_{m}\right) w_{m}=0
$$

where $\left\|w_{m}\right\| \rightarrow \infty$ and so by Lemma 3.1 ,

$$
\left|\psi_{n} k\left(w_{m}\right) w_{m}\right|_{p} /\left\|w_{m}\right\| \rightarrow 0 \quad \text { as } m \rightarrow \infty .
$$

This implies that $\left|L\left(\lambda_{m}\right) v_{m}\right|_{p} /\left\|v_{m}\right\| \stackrel{m}{\rightarrow} 0$. Since $v_{m} L\left(\lambda_{m}\right) v_{m} \leqslant 0$ by (3.18), Lemma 3.6(ii) yields $\lambda=\Lambda(\alpha)$.

\section{Global bifurcation for the truncated problem}

Our goal here is to establish that there is global bifurcation from the trivial solution $v=0$ at $\lambda=\Lambda(\alpha)$ for the inverted truncated equation $F_{n}(\lambda, v)=0$ by using Theorem A.1. We use some of the notation introduced there. As in $\S 3$, we suppose that the conditions (F1), (F2) and (G1) are satisfied with $\Gamma<\alpha<\xi_{1}$ and $p \in\left(\frac{1}{2} N, \infty\right) \cap[2, \infty)$. Clearly,

$$
L(\lambda) u=-\Delta u-\alpha u+\lambda g u
$$

defines a bounded linear operator from $X=W^{2, p}\left(\mathbb{R}^{N}\right)$ into $Y=L^{p}\left(\mathbb{R}^{N}\right)$ and $L \in C^{\infty}(\mathbb{R}, B(X, Y))$. As we have already noted in Lemma 3.5, it follows from $[\mathbf{1 3}$, Theorem 4.3] that

$$
L(\lambda) \in \Phi_{0}(X, Y) \text { for all } \lambda>\alpha,
$$

and it is also shown in that theorem that

$$
Y=\operatorname{ker} L(\lambda) \oplus \operatorname{rge} L(\lambda) \text { for all } \lambda>\alpha .
$$

We already have enough information to enable us to apply the global bifurcation theorem, Theorem A.1, to the inverted truncated problem, but before doing so we 
establish one further result that will help us to obtain a much sharper form of the conclusion.

LEMmA 4.1. For each $n \in \mathbb{N}$, there exists an open neighbourhood $U$ of $(\Lambda(\alpha), 0)$ in $\mathbb{R} \times X$ such that, for all $(\lambda, u) \in U \cap \mathcal{Z}_{n}$, we have $u^{2}>0$ on $\mathbb{R}^{N}$, where $\mathcal{Z}_{n}$ is defined by (3.7).

Proof. We argue by contradiction. If the conclusion is false, then there exists a sequence $\left\{\left(\lambda_{i}, u_{i}\right)\right\} \subset \mathcal{Z}_{n}$ such that $\lambda_{i} \stackrel{i}{\rightarrow} \Lambda(\alpha)$ and $\left\|u_{i}\right\| \stackrel{i}{\rightarrow} 0$ and we can choose this sequence so that for every $i \in \mathbb{N}$, the continuous function $u_{i}$ has at least one zero in $\mathbb{R}^{N}$. We now show that this leads to a contradiction.

Setting $z_{i}=u_{i} /\left\|u_{i}\right\|$, we have

$$
L\left(\lambda_{i}\right) z_{i}+\frac{K_{n}\left(u_{i}\right)}{\left\|u_{i}\right\|}=0 \quad \text { on } \mathbb{R}^{N},
$$

and so

$$
L(\Lambda(\alpha)) z_{i}=\left\{\Lambda(\alpha)-\lambda_{i}\right\} g z_{i}-\frac{K_{n}\left(u_{i}\right)}{\left\|u_{i}\right\|} \stackrel{i}{\rightarrow} 0 \quad \text { in } Y
$$

by Lemma 3.2. On the other hand, by passing to a subsequence we may suppose that $z_{i} \stackrel{i}{\rightarrow} z$ weakly in $X$. Since $L(\Lambda(\alpha)) \in \Phi_{0}(X, Y)$, Lemma 3.5 implies that $z_{i} \stackrel{i}{\rightarrow} z$ strongly in $X$. This means that $\|z\|=1$ and that $L(\Lambda(\alpha)) z=0$. By Lemma 3.6 and Remark 3.1, we have $z \in H^{1}\left(\mathbb{R}^{N}\right)$ and so it follows from Proposition 2.2(ii) that $z^{2}>0$ on $\mathbb{R}^{N}$. Recalling that $X \subset C\left(\mathbb{R}^{N}\right)$, we suppose first that $z>0$ on $\mathbb{R}^{N}$. Since $\Lambda(\alpha)>\alpha$, there exists $i_{0} \in \mathbb{N}$ such that $\lambda_{i} \geqslant \frac{1}{2}\{\Lambda(\alpha)+\alpha\}$ for all $i \geqslant i_{0}$. Setting

$$
\varepsilon=\frac{\Lambda(\alpha)-\alpha}{2\{\Lambda(\alpha)+\alpha\}}
$$

we deduce that there exists $R \geqslant n$ such that $g(x) \geqslant 1-\varepsilon$ for all $|x| \geqslant R$ and hence

$$
-\alpha+\lambda_{i} g \geqslant-\alpha+\frac{1}{2}\{\Lambda(\alpha)+\alpha\}(1-\varepsilon)=\frac{1}{4}\{\Lambda(\alpha)-\alpha\}>0
$$

for all $|x| \geqslant R$ and all $i \geqslant i_{0}$. Since $z_{i} \stackrel{i}{\rightarrow} z$ strongly in $X$ and $\delta=\inf _{|x| \leqslant R} z(x)>0$, it follows that there exists $i_{1} \geqslant i_{0}$ such that $z_{i}(x) \geqslant \frac{1}{2} \delta$ for all $|x| \leqslant R$ and $i \geqslant i_{1}$. But, since $R \geqslant n$, for $|x|>R$, we have

$$
0=L\left(\lambda_{i}\right) z_{i}=-\Delta z_{i}+\left\{-\alpha+\lambda_{i} g\right\} z_{i}
$$

where $z_{i} \geqslant \frac{1}{2} \delta$ for $|x|=R$ and $\lim _{|x| \rightarrow \infty} z_{i}(x)=0$. The weak maximum principle [10, Theorem 8.1] now implies that $z \geqslant 0$ in the region $|x| \geqslant R$, and the strong maximum principle [10, Theorem 8.19] shows that in fact we must have $z_{i}>0$ in the region $|x| \geqslant R$ for all $i \geqslant i_{1}$. Thus $u_{i}>0$ on $\mathbb{R}^{N}$ for all $i \geqslant i_{1}$.

A similar argument shows that $u_{i}<0$ on $\mathbb{R}^{N}$ for all large $i$ in the case where $z<0$.

We now come to the main result of this section. In it we consider the set $\mathcal{Z}_{n}$ of non-trivial solutions of $F_{n}(\lambda, u)=0$ defined in (3.7) with the metric inherited from $\mathbb{R} \times X$.

THEOREM 4.2. Let $\mathcal{C}_{n}$ denote the connected component of $\mathcal{Z}_{n} \cup\{(\Lambda(\alpha), 0)\}$ containing the point $(\Lambda(\alpha), 0)$. Then: 
(a) $u^{2}>0$ on $\mathbb{R}^{N}$ and $\lambda \leqslant \Lambda(\alpha)$ for all $(\lambda, u) \in \mathcal{C}_{n} \backslash\{(\Lambda(\alpha), 0)\}$;

(b) for any $\mu>\alpha$ there exist $T>0$ and $N_{\mu} \in \mathbb{N}$ such that, for all $n \geqslant N_{\mu}$,

$$
\inf P \mathcal{C}_{n}:=\inf \left\{\lambda:(\lambda, u) \in \mathcal{C}_{n}\right\}<\mu \quad \text { and } \quad\|u\| \leqslant T
$$

for all $(\lambda, u) \in \mathcal{C}_{n}$ with $\lambda \geqslant \mu$.

Proof. The first step is to show that Theorem A.1 can be invoked to give some initial information about the global behaviour of $\mathcal{C}_{n}$. Then we use the results of $\S 3$ to deduce that it has the sharper properties claimed above.

From what has already been established in this section, Proposition 2.2(ii) and Lemma 3.2, we can apply Theorem A.1 for $L(\lambda)$ defined by (4.1) on the interval $J=(\alpha, \infty)$ provided that (A.1) holds with $\lambda_{0}=\Lambda(\alpha)$. But $L^{\prime}(\lambda) u=g u$ and, by Lemma 3.6 and Remark 3.1, we have

$$
\operatorname{ker} L(\Lambda(\alpha))=\operatorname{span}\left\{z_{\alpha}\right\} \quad \text { and } \quad L^{\prime}(\Lambda(\alpha)) \operatorname{ker} L(\Lambda(\alpha))=\operatorname{span}\left\{g z_{\alpha}\right\},
$$

where $z_{\alpha}=u_{\Lambda(\alpha)}>0$ on $\mathbb{R}^{N}$ is given by Proposition 2.2(ii). Hence

$$
\operatorname{dim} L^{\prime}(\Lambda(\alpha)) \operatorname{ker} L(\Lambda(\alpha))=1
$$

If $v \in\left[L^{\prime}(\Lambda(\alpha)) \operatorname{ker} L(\Lambda(\alpha))\right] \cap \operatorname{rge} L(\Lambda(\alpha))$, we have

$$
v=\operatorname{tg} z_{\alpha}=L(\Lambda(\alpha)) w \text { on } \mathbb{R}^{N}, \quad \text { for some } t \in \mathbb{R} \text { and } w \in X \text {. }
$$

Furthermore, [13, Theorem 4.1] implies that $w$ and $z_{\alpha} \in W^{2, q}\left(\mathbb{R}^{N}\right)$ for all $q \in$ $(1, \infty)$. Hence

$$
t \int_{\mathbb{R}^{N}} g z_{\alpha}^{2} d x=\int_{\mathbb{R}^{N}}\{L(\Lambda(\alpha)) w\} z_{\alpha} d x=\int_{\mathbb{R}^{N}}\left\{L(\Lambda(\alpha)) z_{\alpha}\right\} w d x=0,
$$

where $\int_{\mathbb{R}^{N}} g z_{\alpha}^{2} d x \neq 0$ since $z_{\alpha}>0$ on $\mathbb{R}^{N}$. This proves that $t=0$ and consequently

$$
\left[L^{\prime}(\Lambda(\alpha)) \operatorname{ker} L(\Lambda(\alpha))\right] \cap \operatorname{rge} L(\Lambda(\alpha))=\{0\} .
$$

By (4.2) and (4.3) we know that codim rge $L(\Lambda(\alpha))=1$, and we have already noted that

$$
\operatorname{dim} L^{\prime}(\Lambda(\alpha)) \operatorname{ker} L(\Lambda(\alpha))=1
$$

This establishes (A.1) and we can assert that $\mathcal{C}_{n}$ satisfies the conclusion of Theorem A.1.

The next step is to show that if $(\lambda, u) \in \mathcal{C}_{n} \backslash\{(\Lambda(\alpha), 0)\}$ then $u$ has no zeros. For this we set

$$
\mathcal{Q}=\left\{(\lambda, u) \in \mathcal{C}_{n}: u^{2}>0 \text { on } \mathbb{R}^{N}\right\} \cup\{(\Lambda(\alpha), 0)\}
$$

and prove that $\mathcal{Q}=\mathcal{C}_{n}$ by showing that $\mathcal{Q}$ is both an open and closed subset of $\mathcal{C}_{n}$.

First we prove that $\mathcal{Q}$ is open in $\mathcal{C}_{n}$. Given $(\lambda, u) \in \mathcal{Q}$, we must show that there exists an open neighbourhood $U$ of $(\lambda, u)$ in $\mathbb{R} \times X$ such that $U \cap \mathcal{C}_{n} \subset \mathcal{Q}$. For $(\lambda, u)=(\Lambda(\alpha), 0)$ this is established in Lemma 4.1 , so we can suppose that $(\lambda, u) \in \mathcal{C}_{n}$ with $u^{2}>0$ on $\mathbb{R}^{N}$. Since $X \subset C\left(\mathbb{R}^{N}\right)$, this means that $u$ does not change sign on $\mathbb{R}^{N}$. Let us suppose that $u>0$ on $\mathbb{R}^{N}$, the case $u<0$ being similar. As in the proof of Lemma 4.1, since $\lambda>\alpha$ and $\lim _{|x| \rightarrow \infty} g(x)=1$, there exist $\eta>0$ and $R>0$ such that for all $\mu$ with $|\lambda-\mu| \leqslant \eta$,

$$
-\alpha+\mu g>0 \text { for all }|x| \geqslant R \text {. }
$$


Let $\delta=\inf _{|x| \leqslant R} u(x)$. Since $\delta>0$, there exists a neighbourhood $U$ of $(\lambda, u)$ in $\mathbb{R} \times X$ such that $|\lambda-\mu| \leqslant \eta$ and $\inf _{|x| \leqslant R} v(x) \geqslant \frac{1}{2} \delta$ for all $(\mu, v) \in U$. In particular, $v \Delta v \geqslant 0$ for $|x| \geqslant R, v>0$ when $|x|=R$ and $\lim _{|x| \rightarrow \infty} v(x)=0$, for $(\mu, v) \in U \cap \mathcal{Z}_{n}$. Therefore, as in the proof of Lemma 4.1, the maximum principle implies that $v>0$ when $|x|>R$, too. Hence $U \cap \mathcal{C}_{n} \subset \mathcal{Q}$ and $\mathcal{Q}$ is open.

Now we show that $\mathcal{Q}$ is closed in $\mathcal{C}_{n}$. Suppose that $(\lambda, u) \in \mathcal{C}_{n}$ and that there exists a sequence $\left\{\left(\lambda_{i}, u_{i}\right)\right\} \subset \mathcal{Q}$ such that $\lambda_{i} \stackrel{i}{\rightarrow} \lambda$ and $\left\|u_{i}-u\right\| \stackrel{i}{\rightarrow} 0$. If $u=0$, then $\lambda=\Lambda(\alpha)$ since $\mathcal{C}_{n} \cap[\mathbb{R} \times\{0\}]=\{(\Lambda(\alpha), 0)\}$ and so $(\lambda, u) \in \mathcal{Q}$. Consider now $u \neq 0$. We have $L(\lambda) u+K_{n}(u)=0$ and, passing to a subsequence, we can suppose that either $u_{i}>0$ for all $i \in \mathbb{N}$ or $u_{i}<0$ for all $i \in \mathbb{N}$. In the first case it follows that $u \geqslant 0$ on $\mathbb{R}^{N}$ and

$$
-\Delta u+c_{+} u=c_{-} u \geqslant 0 \quad \text { on } \mathbb{R}^{N}
$$

where $c=-\alpha+\lambda g+\psi_{n} k\left(u /\|u\|^{2}\right)$, and the strong maximum principle [10, Theorem 8.19] shows that either $u \equiv 0$ or $u>0$ on $\mathbb{R}^{N}$. Hence $(\lambda, u) \in \mathcal{Q}$. In the case where $u_{i}<0$ for all $i \in \mathbb{N}$, a similar argument shows that either $u \equiv 0$ or $u<0$ on $\mathbb{R}^{N}$. Thus, in all cases $(\lambda, u) \in \mathcal{Q}$ and $\mathcal{Q}$ is closed in $\mathcal{C}_{n}$.

We have shown that $\mathcal{C}_{n}=\mathcal{Q}$ and we claim that this means that case (ii) in Theorem A.1 cannot occur. Indeed, if $\mathcal{C}_{n}$ has the property (ii), there exist $\lambda \in$ $J \backslash\{\Lambda(\alpha)\}$ and a sequence $\left\{\left(\lambda_{i}, u_{i}\right)\right\} \subset \mathcal{C}_{n}$ such that $\lambda_{i} \stackrel{i}{\rightarrow} \lambda$ and $\left\|u_{i}\right\| \stackrel{i}{\rightarrow} 0$. We can suppose that $\left\|u_{i}\right\|>0$ since $\mathcal{C}_{n} \cap[\mathbb{R} \times\{0\}]=\{(\Lambda(\alpha), 0)\}$. Setting $z_{i}=u_{i} /\left\|u_{i}\right\|$ and arguing as in the proof of Lemma 4.1, we see that, by passing to a subsequence, we may assume that $z_{i} \stackrel{i}{\rightarrow} z$ in $X$ and that $L(\lambda) z=0$ with $\|z\|=1$. By [13, Corollary 4.1] with $V=\lambda g$, we can deduce that $z \in H^{1}\left(\mathbb{R}^{N}\right)$ and satisfies (2.2). It follows from Proposition 2.2(ii) that $\lambda<\Lambda(\alpha)$, and $z$ changes sign on $\mathbb{R}^{N}$. But since $\left\{\left(\lambda_{i}, u_{i}\right)\right\} \in \mathcal{Q} \backslash\{(\Lambda(\alpha), 0)\}$ the sequence $\left\{z_{i}\right\}$ can be chosen so that either $z_{i}>0$ on $\mathbb{R}^{N}$ for all $i \in \mathbb{N}$ or $z_{i}<0$ on $\mathbb{R}^{N}$ for all $i \in \mathbb{N}$. In the first case we have $z \geqslant 0$ on $\mathbb{R}$, and in the second $z \leqslant 0$ on $\mathbb{R}$, which contradicts the earlier conclusion.

We now know that $\mathcal{C}_{n}$ has at least one of the properties (i) and (iii) of Theorem A.1. If $(\lambda, u) \in \mathcal{C}_{n} \backslash\{(\Lambda(\alpha), 0)\}$, we have

$$
-\Delta u-\alpha u+\lambda g u+\psi_{n} k\left(\frac{u}{\|u\|^{2}}\right) u=0 \quad \text { and } \quad u^{2}>0 \quad \text { on } \mathbb{R}^{N} \text {. }
$$

By [13, Corollary 4.1] with $V=\lambda g+\psi_{n} k\left(u /\|u\|^{2}\right)$, we have $u \in W^{2, q}\left(\mathbb{R}^{N}\right)$ for all $q \in(1, \infty)$. Hence, using Proposition 2.2, we have

$$
\begin{aligned}
0 & =\inf \left\{a_{\Lambda(\alpha)}(v): v \in H^{1}\left(\mathbb{R}^{N}\right)\right\} \\
& \leqslant a_{\Lambda(\alpha)}(u)=\int_{\mathbb{R}^{N}}\left(|\nabla u|^{2}-\alpha u^{2}+\Lambda(\alpha) g u^{2}\right) d x \\
& \leqslant \int_{\mathbb{R}^{N}}\left(|\nabla u|^{2}-\alpha u^{2}+\Lambda(\alpha) g u^{2}+\psi_{n} k\left(\frac{u}{\|u\|^{2}}\right) u^{2}\right) d x \\
& =\{\Lambda(\alpha)-\lambda\} \int_{\mathbb{R}^{N}} g u^{2} d x
\end{aligned}
$$

where $\int_{\mathbb{R}^{N}} g u^{2} d x>0$ since $u^{2}>0$ on $\mathbb{R}^{N}$. Thus

$$
\lambda \leqslant \Lambda(\alpha) \text { for all }(\lambda, u) \in \mathcal{C}_{n} \backslash\{(\Lambda(\alpha), 0)\} .
$$

Now consider some $\mu>\alpha$. By Lemma 3.3(ii), there exists $N_{\mu} \in \mathbb{N}$ such that $\|u\| \leqslant T$ for all $(\lambda, u) \in \mathcal{C}_{n}$ with $\lambda \geqslant \mu$ and $n \geqslant N_{\mu}$. Thus, if $n \geqslant N_{\mu}$ and 
$\inf \mathcal{C}_{n} \geqslant \mu$, the component $\mathcal{C}_{n}$ is bounded in $\mathbb{R} \times X$ and $\inf \mathcal{C}_{n}>\alpha=\inf J$, which contradicts Theorem A.1. Hence $\inf \mathcal{C}_{n}<\mu$ for all $n \geqslant N_{\mu}$.

\section{Proof of Theorem 2.3}

We suppose throughout this section that the conditions (F1), (F2) and (G1) are satisfied with $\Gamma<\alpha<\xi_{1}$ and we fix $p \in[2, \infty) \cap\left(\frac{1}{2} N, \infty\right)$.

LEMMA 5.1. If $(\lambda, u)$ is a solution of problem (1.1), then $\lambda<\Lambda(\alpha)$.

Proof. If $(\lambda, u)$ is a solution of problem (1.1), then

$$
\int_{\mathbb{R}^{N}}\left(|\nabla u|^{2}-\alpha u^{2}+\lambda g(x) u^{2}+k(u) u^{2}\right) d x=0
$$

where $k$ is given by (1.6). Recalling Proposition 2.2(iv), we have

$$
\begin{aligned}
0 & =\inf \left\{a_{\Lambda(\alpha)}(v): v \in H^{1}\left(\mathbb{R}^{N}\right)\right\} \\
& \leqslant a_{\Lambda(\alpha)}(u)=(\Lambda(\alpha)-\lambda) \int_{\mathbb{R}^{N}} g u^{2} d x-\int_{\mathbb{R}^{N}} k(u) u^{2} d x .
\end{aligned}
$$

Hence,

$$
0 \leqslant \int_{\mathbb{R}^{N}} k(u) u^{2} d x \leqslant(\Lambda(\alpha)-\lambda) \int_{\mathbb{R}^{N}} g u^{2} d x .
$$

But, in fact, $\int_{\mathbb{R}^{N}} k(u) u^{2} d x>0$ since $u \in C\left(\mathbb{R}^{N}\right), u \not \equiv 0, \lim _{|x| \rightarrow \infty} u(x)=0$ and $k(0)=\alpha+1$. Hence $\lambda<\Lambda(\alpha)$.

By using the global bifurcation results for the truncated problem $F_{n}(\lambda, v)=0$ (see Theorem 4.2), we prove first the following bifurcation result for the inverted problem $F(\lambda, v)=0$, and then the asymptotic bifurcation Theorem 2.3 , for $G(\lambda, u)=0$, that is, (1.1).

Let $X$ be as in $\S 3$ and consider $\tilde{\mathcal{Z}} \cup\{(\Lambda(\alpha), 0)\}$ as a metric space with the metric inherited from $\mathbb{R} \times X$ where $\tilde{\mathcal{Z}}$ is defined below.

THEOREM 5.2. Let $U$ be an open and bounded subset of $\mathbb{R} \times X$ such that $(\Lambda(\alpha), 0) \in U$ with

$$
\mu:=\inf \{\lambda:(\lambda, u) \in U\}>\alpha .
$$

Let $\tilde{\mathcal{Z}}=\left\{(\lambda, v) \in \mathcal{Z}: v^{2}>0\right.$ on $\left.\mathbb{R}^{N}\right\}$, where $\mathcal{Z}$ is given by (3.8). The following hold.

(i) We have $\tilde{\mathcal{Z}} \cap \partial U \neq \emptyset$.

(ii) Let $\mathcal{C}$ be the connected component of $\tilde{\mathcal{Z}} \cup\{(\Lambda(\alpha), 0)\}$ containing $(\Lambda(\alpha), 0)$. Then $\mathcal{C}$ is bounded with inf $P \mathcal{C}=\alpha$ and $\sup P \mathcal{C}=\Lambda(\alpha)$, where $P(\lambda, v)=\lambda$ for all $(\lambda, v) \in \mathbb{R} \times X$. In fact, $P\{\mathcal{C} \backslash\{(\Lambda(\alpha), 0)\}\}=(\alpha, \Lambda(\alpha))$.

(iii) If $\left\{\left(\lambda_{n}, v_{n}\right)\right\} \subset \mathcal{C}$ with $\lambda_{n} \stackrel{n}{\rightarrow} \lambda>\alpha$ and $\lim _{n \rightarrow \infty}\left\|v_{n}\right\|=0$, then $\lambda=\Lambda(\alpha)$. Furthermore, if $\left\{\left(\lambda_{n}, v_{n}\right)\right\} \subset \mathcal{C}$ with $\lim _{n \rightarrow \infty} \lambda_{n}=\Lambda(\alpha)$ then $\lim _{n \rightarrow \infty}\left\|v_{n}\right\|=0$.

Proof. (i) By Theorem 4.2, since $\mu>\alpha$ there exists $N_{\mu} \in \mathbb{N}$ such that

$$
\mathcal{C}_{n} \cap \partial U \neq \emptyset \text { for all } n \geqslant N_{\mu},
$$

where $\mathcal{C}_{n}$ is the connected component of $\mathcal{Z}_{n} \cup\{(\Lambda(\alpha), 0)\}$ containing $\{(\Lambda(\alpha), 0)\}$. 
Let $\left(\lambda_{n}, v_{n}\right) \in \mathcal{C}_{n} \cap \partial U$ for all $n \geqslant N_{\mu}$. Then, by Theorem 4.2(a) we have

$$
\alpha<\mu \leqslant \lambda_{n} \leqslant \Lambda(\alpha) \text { and } v_{n}^{2}>0 .
$$

By passing to a subsequence we may suppose that either $v_{n}>0$ or $v_{n}<0$ for all $n \geqslant N_{\mu}$ since $v_{n} \in C\left(\mathbb{R}^{N}\right)$, and

$$
\begin{aligned}
& \lambda_{n} \stackrel{n}{\rightarrow} \lambda \in[\mu, \Lambda(\alpha)], \\
& v_{n} \stackrel{n}{\rightarrow} v \text { weakly in } X \text { with } v \geqslant 0 \text { or } v \leqslant 0, \\
& \left\|v_{n}\right\| \stackrel{n}{\rightarrow} \ell \geqslant 0 .
\end{aligned}
$$

We claim that $\ell>0$. In fact, if $\ell=0$ we have

$$
\left(\lambda_{n}, v_{n}\right) \stackrel{n}{\rightarrow}(\lambda, 0) \in \partial U
$$

Consequently, $\lambda \neq \Lambda(\alpha)$.

On the other hand, it follows from $\left(\lambda_{n}, v_{n}\right) \in \mathcal{Z}_{n}$ that

$$
L\left(\lambda_{n}\right) v_{n}+\psi_{n} k\left(\frac{v_{n}}{\left\|v_{n}\right\|^{2}}\right) v_{n}=0
$$

and this implies that $v_{n} L\left(\lambda_{n}\right) v_{n} \leqslant 0$ since $\psi_{n} \geqslant 0$ and $k(s) \geqslant 0$. By Lemma 3.6(i) we know that

$$
\left|k\left(\frac{v_{n}}{\left\|v_{v}\right\|^{2}}\right) v_{n}\right|_{p} /\left\|v_{n}\right\| \stackrel{n}{\rightarrow} 0
$$

Then it follows from (5.3) that

$$
\left|L\left(\lambda_{n}\right) v_{n}\right|_{p} /\left\|v_{n}\right\|=\left|-\psi_{n} k\left(\frac{v_{n}}{\left\|v_{v}\right\|^{2}}\right) v_{n}\right|_{p} /\left\|v_{n}\right\| \leqslant\left|k\left(\frac{v_{n}}{\left\|v_{v}\right\|^{2}}\right) v_{n}\right|_{p} /\left\|v_{n}\right\| \stackrel{n}{\rightarrow} 0 .
$$

So, $\lambda=\Lambda(\alpha)$ by Lemma 3.6(ii), contradicting our earlier conclusion. Hence $\ell>0$.

Next, we show that

$$
\left\|v_{n}-v\right\| \stackrel{n}{\rightarrow} 0 .
$$

For any $R>0$, the compactness of Sobolev embeddings implies that

$$
\frac{v_{n}}{\left\|v_{n}\right\|^{2}} \stackrel{n}{\rightarrow} \frac{v}{\ell^{2}} \quad \text { uniformly on } B_{R}:=\left\{x \in \mathbb{R}^{N}:|x| \leqslant R\right\} \text {. }
$$

So,

$$
k\left(\frac{v_{n}}{\left\|v_{n}\right\|^{2}}\right) v_{n} \stackrel{n}{\rightarrow} k\left(\frac{v}{\ell^{2}}\right) v \quad \text { uniformly on } B_{R}
$$

Therefore,

$$
\psi_{n} k\left(\frac{v_{n}}{\left\|v_{n}\right\|^{2}}\right) v_{n} \stackrel{n}{\rightarrow} k\left(\frac{v}{\ell^{2}}\right) v \quad \text { uniformly on } B_{R} .
$$

By (1.6), $\left|\psi_{n} k(s)\right| \leqslant \alpha+1$, so using Lemma 3.4 and dominated convergence, we easily deduce that

$$
\psi_{n} k\left(\frac{v_{n}}{\left\|v_{n}\right\|^{2}}\right) v_{n} \stackrel{n}{\rightarrow} k\left(\frac{v}{\ell^{2}}\right) v \text { in } L^{p}\left(\mathbb{R}^{N}\right) \quad \text { for all } 2 \leqslant p<\infty .
$$

Using (5.3), we now have

$$
\begin{gathered}
L(\lambda) v_{n}=-\left(\lambda_{n}-\lambda\right) g v_{n}-\psi_{n} k\left(\frac{v_{n}}{\left\|v_{n}\right\|^{2}}\right) v_{n} \\
\stackrel{n}{\rightarrow}-k\left(\frac{v}{\ell^{2}}\right) v \text { in } Y, \quad \text { by }(5.5) .
\end{gathered}
$$


Since $\lambda \geqslant \mu>\alpha$, we have $L(\lambda) \in \Phi_{0}(X, Y)$ and Lemma 3.5 yields $v_{n} \stackrel{n}{\rightarrow} v$ strongly in $X$, proving (5.4).

Therefore, $\lim _{n \rightarrow \infty}\left\|v_{n}\right\|=\|v\|=\ell>0,(\lambda, v) \in \partial U$, and

$$
L(\lambda) v=-k\left(\frac{v}{\|v\|^{2}}\right) v, \quad \text { that is, } \quad F(\lambda, v)=0 .
$$

We now claim that $v^{2}>0$ on $\mathbb{R}^{N}$. Indeed, by (5.2) we know that either $v \geqslant 0$ on $\mathbb{R}^{N}$ or $v \leqslant 0$ on $\mathbb{R}^{N}$. Suppose that $v \geqslant 0$ on $\mathbb{R}^{N}$. It follows from (5.7) that

$$
-\Delta v+c_{+} v=c_{-} v \geqslant 0,
$$

where $c:=-\alpha+\lambda g+k\left(v /\|v\|^{2}\right)$ and $c_{ \pm}=\max \{ \pm c, 0\}$. Since $\lim _{|x| \rightarrow \infty} v(x)=0$, with $v \not \equiv 0$, the strong maximum principle $\left[\mathbf{1 0}\right.$, Theorem 8.19] gives $v>0$ on $\mathbb{R}^{N}$. Similarly, if $v \leqslant 0$ on $\mathbb{R}^{N}$, then we have $v<0$ on $\mathbb{R}^{N}$. Thus, $v^{2}>0$ and $(\lambda, v) \in \tilde{\mathcal{Z}}$, so that $\tilde{\mathcal{Z}} \cap \partial U \neq \emptyset$.

(ii) Let $\mathcal{Q}=\tilde{\mathcal{Z}} \cup\{(\Lambda(\alpha), 0)\}$ and let $\mathcal{C}$ be the connected component of $\mathcal{Q}$ containing $(\Lambda(\alpha), 0)$.

For any $(\lambda, v) \in \mathcal{Z}$, we have $F(\lambda, v)=0$ with $v \not \equiv 0$. Then it is easy to see that $(\lambda, u)$ with $u=v /\|v\|^{2}$ is a solution of $G(\lambda, u)=0$. Since $\lambda>\alpha$, it follows from Lemma 3.4 and Remark 3.1 that $(\lambda, u)$ is a solution of (1.1) and hence by Lemma 5.1, we have $\lambda<\Lambda(\alpha)$. In particular, $\{\lambda:(\lambda, v) \in \mathcal{C} \backslash\{(\Lambda(\alpha), 0)\}\} \subset(\alpha, \Lambda(\alpha))$ and hence $\sup P \mathcal{C}=\Lambda(\alpha)$. Therefore, it follows from Lemma 3.3(i) that $\mathcal{Z}$ is bounded in $\mathbb{R} \times X$, as are $\tilde{\mathcal{Z}}, \mathcal{Q}$ and $\mathcal{C}$. Suppose that

$$
\mu:=\inf P \mathcal{C}>\alpha
$$

and note that $\mu \leqslant \Lambda(\alpha)$. For any $\bar{\mu} \in(\alpha, \mu)$, let

$$
\begin{gathered}
V=\{(\lambda, v) \in \mathcal{Q}: \lambda \in[\bar{\mu}, \Lambda(\alpha)]\}=\{(\lambda, v) \in \mathcal{Q}: \lambda \geqslant \bar{\mu}\}, \\
V_{1}=\{(\Lambda(\alpha), 0)\} \quad \text { and } \quad V_{2}=\mathcal{Q} \cap[\{\bar{\mu}\} \times X]=\{(\lambda, v) \in \mathcal{Q}: \lambda=\bar{\mu}\} .
\end{gathered}
$$

By Lemma C.1, $V$ is a compact subset of $\mathbb{R} \times X$. We now deduce from a result of Whyburn [26] (see Lemma C.2) that there exists a connected subset $V_{0}$ of $V$ such that

$$
V_{0} \cap V_{1} \neq \emptyset \quad \text { and } \quad V_{0} \cap V_{2} \neq \emptyset .
$$

In fact, if there is no connected subset of $V$ such that (5.9) holds, then by Lemma C.2 there must exist compact subsets $U_{1}$ and $U_{2}$ of $V$ such that

$$
V=U_{1} \cup U_{2}, \quad V_{1} \subset U_{1}, \quad V_{2} \subset U_{2}, \quad U_{1} \cap U_{2}=\emptyset .
$$

So, there exists $\delta>0$ such that $\operatorname{dist}\left(U_{1}, U_{2}\right)=2 \delta$. Let

$$
W_{\delta}=\left\{x \in \mathbb{R} \times X: \operatorname{dist}\left(x, U_{1}\right)<\delta\right\} \cap\{(\bar{\mu}, \infty) \times X\},
$$

which is an open bounded set in $\mathbb{R} \times X$. Furthermore, $\bar{W}_{\delta} \cap U_{2}=\emptyset$. Clearly, $\inf P W_{\delta} \geqslant \bar{\mu}>\alpha$. Then $\tilde{\mathcal{Z}} \cap \partial W_{\delta} \neq \emptyset$ by part (i). However, for any $(\lambda, v) \in \tilde{\mathcal{Z}} \cap \partial W_{\delta}$ we have $\bar{\mu} \leqslant \lambda<\Lambda(\alpha)$ and $(\lambda, v) \in \mathcal{Q}$, so that $(\lambda, v) \in V=U_{1} \cup U_{2}$. But if $(\lambda, v) \in \partial W_{\delta}$, then $(\lambda, v) \in \bar{W}_{\delta}$ and $(\lambda, v) \notin U_{2}$. This implies that $(\lambda, v) \in U_{1}$ which is impossible since $(\lambda, v) \in \partial W_{\delta}$. So, (5.9) is proved.

By (5.9), it is obvious that inf $P V_{0}=\bar{\mu}<\mu$. But $V_{0} \subset \mathcal{C}$ and so inf $P \mathcal{C} \leqslant \bar{\mu}<\mu$, a contradiction. Hence inf $P \mathcal{C}=\alpha$.

(iii) Let $\left\{\left(\lambda_{n}, v_{n}\right)\right\} \subset \mathcal{C} \backslash\{(\Lambda(\alpha), 0)\}$ be such that $\lambda_{n} \rightarrow \lambda>\alpha$ and $\left\|v_{n}\right\| \stackrel{n}{\rightarrow} 0$. Then $v_{n}^{2}>0$ and $v_{n} L\left(\lambda_{n}\right) v_{n} \leqslant 0$ so it follows from Lemma 3.6 that $\lambda=\Lambda(\alpha)$. 
On the other hand, suppose that there exists a sequence

$$
\left\{\left(\lambda_{n}, v_{n}\right)\right\} \subset \mathcal{C} \backslash\{(\Lambda(\alpha), 0)\}
$$

such that $\lambda_{n} \stackrel{n}{\rightarrow} \Lambda(\alpha)$ and $\left\|v_{n}\right\| \geqslant \delta>0$ for all $n \in \mathbb{N}$. Since $\left\|v_{n}\right\| \leqslant T$ by Lemma 3.3, passing to a further subsequence, we may assume that $v_{n} \stackrel{n}{\rightarrow} v$ weakly in $X$. Then repeating the proof of (5.4), just replacing $\psi_{n}(x)$ by 1 , we find that $v_{n} \stackrel{n}{\rightarrow} v$ strongly in $X$ and $F(\Lambda(\alpha), v)=0$. Thus $(\Lambda(\alpha), v) \in \mathcal{Z}$, whereas, at the beginning of the proof of part (ii) we showed that $\lambda<\Lambda(\alpha)$ for all $(\lambda, v) \in \mathcal{Z}$. Thus $\left\|v_{n}\right\| \rightarrow 0$ if $\lambda_{n} \stackrel{n}{\rightarrow} \Lambda(\alpha)$.

Theorem 5.2 establishes the global properties of a connected subset of $\tilde{\mathcal{Z}} \cup\{(\Lambda(\alpha), 0)\}$. However, in order to maintain connectedness under inversion, we need to find a connected subset of $\tilde{\mathcal{Z}}$ having similar properties. This can be achieved by a procedure due to J. C. Alexander [1].

Corollary 5.3. Let $\tilde{\mathcal{Z}}$ and $\mathcal{C}$ be as given in Theorem 5.2. Then there exists a bounded connected subset $\mathcal{C}_{0}$ of $\tilde{\mathcal{Z}}$ such that inf $P \mathcal{C}_{0}=\alpha$ and $(\Lambda(\alpha), 0) \in \overline{\mathcal{C}}_{0}$. In particular, $\sup P \mathcal{C}_{0}=\Lambda(\alpha)$ and $0<\|v\| \leqslant T$ for all $(\lambda, v) \in \mathcal{C}_{0}$.

Proof. Recall that $\mathcal{C}$ is bounded in $\mathbb{R} \times X$ with $0 \leqslant\|v\| \leqslant T$ for all $(\lambda, v) \in \mathcal{C}$ by Lemma 3.3, and that there exists a sequence $\left\{\left(\lambda_{n}, v_{n}\right)\right\} \subset \mathcal{C}$ with $\lambda_{n} \stackrel{n}{\rightarrow} \alpha$. Let $\mathcal{T}$ denote the topology on $\mathcal{C}$, that is,

$$
\mathcal{T}=\{\mathcal{C} \cap W: W \text { is an open subset of } \mathbb{R} \times X\} .
$$

We now make a one-point compactification of $\mathcal{C}$ by adding a 'point at $\alpha$ ' to $\mathcal{C}$ as follows:

$$
\mathcal{C}^{\infty}:=\mathcal{C} \cup\{\infty\}
$$

and we define a topology $\mathcal{T}^{\infty}$ on $\mathcal{C}^{\infty}$ as follows:

$$
A \in \mathcal{T}^{\infty} \Longleftrightarrow\left\{\begin{array}{l}
A \in \mathcal{T} \text { if } \infty \notin A ; \\
\text { or } \\
A \text { is the union of an element in } \mathcal{T} \text { and a set of the form } \\
\mathcal{N}_{\beta} \cup\{\infty\} \text { for some } \beta \in(\alpha, \Lambda(\alpha)] \text { if } \infty \in A,
\end{array}\right.
$$

where $\mathcal{N}_{\beta}=\{\mathcal{C} \cap[(\alpha, \beta) \times X]: \beta \in(\alpha, \Lambda(\alpha)]\}$.

It is not difficult to verify that $\mathcal{T}^{\infty}$ is indeed a topology in $\mathcal{C}^{\infty}$ and we claim that $\left(\mathcal{C}^{\infty}, \mathcal{T}^{\infty}\right)$ is a compact topological space. In fact, let $\left\{A_{i}\right\}$ be an open cover of $\mathcal{C}^{\infty}$. Then there exists $i_{0}$ such that $\infty \in A_{i_{0}}$ with $A_{i_{0}}=B_{i_{0}} \cup C_{i_{0}}$, where $B_{i_{0}} \in \mathcal{T}$ and $C_{i_{0}}=\mathcal{N}_{\beta_{1}} \cup\{\infty\}$ for some $\beta_{1} \in(\alpha, \Lambda(\alpha)]$. Note that $\mathcal{C} \subset \bigcup_{i=1}^{\infty}\left(A_{i} \backslash\{\infty\}\right)$ and $A_{i} \backslash\{\infty\} \in \mathcal{T}$. Setting $\gamma=\frac{1}{2}\left(\alpha+\beta_{1}\right)$, we see from Lemma C.1 that $\{[\gamma, \Lambda(\alpha)] \times X\} \cap \mathcal{C}$ is compact and is also covered by $A_{i} \backslash\{\infty\}$. So, there exists a finite subcover $A_{i_{j}}$, for $j=1,2, \ldots, k$, such that $\{[\gamma, \Lambda(\alpha)] \times X\} \cap \mathcal{C} \subset \bigcup_{j=1}^{k} A_{i_{j}}$ and the claim is proved since $\mathcal{C}^{\infty} \subset A_{i_{0}} \cup(\{[\gamma, \Lambda(\alpha)] \times X\} \cap \mathcal{C})$.

The sets $A=\{(\Lambda(\alpha), 0)\}$ and $B=\{\infty\}$ are closed in $\mathcal{C}^{\infty}$. If $A$ and $B$ are separated in $\mathcal{C}^{\infty}$, then there exist $V_{A}$ and $V_{B}$ in $\mathcal{T}^{\infty}$ such that

$$
V_{A} \cup V_{B}=\mathcal{C}^{\infty}, \quad V_{A} \cap V_{B}=\emptyset \text { and } A \subset V_{A}, \quad B \subset V_{B} .
$$

Clearly $\infty \notin V_{A}$, so $V_{A} \in \mathcal{T}$. Also $V_{B} \backslash\{\infty\} \in \mathcal{T}$ and it is non-empty. But, $V_{A} \cap\left[V_{B} \backslash\{\infty\}\right]=\emptyset$ and $V_{A} \cup\left[V_{B} \backslash\{\infty\}\right]=\mathcal{C}$, contradicting the connectedness of $\mathcal{C}$. 
Hence, $A$ and $B$ are not separated in $\mathcal{C}^{\infty}$. By Lemma C.3, there exists a connected set $\mathcal{C}_{0}$ in $\mathcal{C}^{\infty} \backslash\{A \cup B\}=\mathcal{C} \backslash\{(\Lambda(\alpha), 0)\}$ such that $(\Lambda(\alpha), 0) \in \overline{\mathcal{C}}_{0}^{\mathcal{T}^{\infty}}$ and $\infty \in \overline{\mathcal{C}}_{0}^{\mathcal{T}^{\infty}}$, where $\overline{\mathcal{C}}_{0}^{\mathcal{T}^{\infty}}$ denotes the closure of $\mathcal{C}_{0}$ in $\left(\mathcal{C}^{\infty}, \mathcal{T}^{\infty}\right)$. Then $\mathcal{C}_{0}$ is also connected in $(\mathcal{C}, \mathcal{T})$. Otherwise, there are non-empty sets $P, Q \in \mathcal{T}$ such that

$$
P \cap Q=\emptyset \quad \text { and } \quad \mathcal{C}_{0}=P \cup Q .
$$

However, since $P, Q \in \mathcal{T}^{\infty}$, this contradicts the connectedness of $\mathcal{C}_{0}$ in $\mathcal{T}^{\infty}$.

Now, we claim that $(\Lambda(\alpha), 0) \in \overline{\mathcal{C}}_{0}^{\mathcal{T}}$. Indeed, let $U \in \mathcal{T}$ with $(\Lambda(\alpha), 0) \in U$. Clearly, $U \in \mathcal{T}^{\infty}$. Hence it follows from $(\Lambda(\alpha), 0) \in \overline{\mathcal{C}}_{0}^{\mathcal{T}^{\infty}}$ that $U \cap \mathcal{C}_{0} \neq \emptyset$ and $(\Lambda(\alpha), 0) \in \overline{\mathcal{C}}_{0}^{\mathcal{T}}$. This implies that $\sup P \mathcal{C}_{0}=\Lambda(\alpha)$.

Finally, we show that inf $P \mathcal{C}_{0}=\alpha$. For this purpose, it is enough to prove that

$$
\mathcal{C}_{0} \cap\{[(\alpha, \beta) \times X] \cap \mathcal{C}\} \neq \emptyset \quad \text { for any } \beta \in(\alpha, \Lambda(\alpha)] .
$$

In fact, since $\{[(\alpha, \beta) \times X] \cap \mathcal{C}\} \cup\{\infty\} \in \mathcal{T}^{\infty}$ and $\infty \in \overline{\mathcal{C}}_{0}^{\mathcal{T}^{\infty}}$, we have

$$
[\{[(\alpha, \beta) \times X] \cap \mathcal{C}\} \cup\{\infty\}] \cap \mathcal{C}_{0} \neq \emptyset .
$$

But $\infty \neq \mathcal{C}_{0}$ and hence $\{[(\alpha, \beta) \times X] \cap \mathcal{C}\} \cap \mathcal{C}_{0} \neq \emptyset$.

REMARK 5.1. Since $\mathcal{Z} \subset \mathbb{R} \times W^{2, p}\left(\mathbb{R}^{N}\right) \subset \mathbb{R} \times C\left(\mathbb{R}^{N}\right)$, it follows that the set $\mathcal{C}_{0}$ obtained in Corollary 5.3 satisfies either

$$
\mathcal{C}_{0} \subset \mathcal{Z}^{+}:=\left\{(\lambda, v) \in \mathcal{Z}: v>0 \text { on } \mathbb{R}^{N}\right\}
$$

or

$$
\mathcal{C}_{0} \subset \mathcal{Z}^{-}:=\left\{(\lambda, v) \in \mathcal{Z}: v<0 \text { on } \mathbb{R}^{N}\right\}
$$

Corollary 5.4. Suppose that, in addition to the hypotheses of Corollary 5.3, the function $f$ is odd. Then there exist two bounded connected subsets $\mathcal{C}_{0}^{+}$and $\mathcal{C}_{0}^{-}$ of $\mathcal{Z}^{+}$and $\mathcal{Z}^{-}$, respectively, each of which has the same properties as $\mathcal{C}_{0}$.

Proof. It is sufficient to note that

$$
\widetilde{\mathcal{C}_{0}}=\left\{(\lambda,-v):(\lambda, v) \in \mathcal{C}_{0}\right\}
$$

is also a connected subset of $\widetilde{\mathcal{Z}}$ and that either $\mathcal{C}_{0} \subset \mathcal{Z}^{+}$and $\widetilde{\mathcal{C}_{0}} \subset \mathcal{Z}^{-}$or vice versa.

Finally, we can prove our main Theorem 2.3.

Proof of Theorem 2.3. Let $f_{R}$ and $f_{L}$ be the odd functions defined by

$$
f_{R}(s)=\left\{\begin{array}{ll}
f(s) & \text { for } s \geqslant 0, \\
-f(-s) & \text { for } s<0,
\end{array} \quad \text { and } \quad f_{L}(s)= \begin{cases}-f(-s) & \text { for } s \geqslant 0 \\
f(s) & \text { for } s<0\end{cases}\right.
$$

Both of these functions satisfy the hypotheses (F1) and (F2) and so Corollary 5.4 is valid with $f$ replaced by $f_{R}$ and $f_{L}$. Let $\mathcal{C}_{0}^{+}$and $\mathcal{C}_{0}^{-}$be the connected subsets of positive or negative solutions for the problem with $f_{R}$ or $f_{L}$, respectively. Then we have $\mathcal{C}_{0}^{ \pm} \subset \mathcal{Z}^{ \pm}$for $f$ with

$$
\inf P \mathcal{C}_{0}^{ \pm}=\alpha \quad \text { and } \quad(\Lambda(\alpha), 0) \in \overline{\mathcal{C}_{0}^{ \pm}} .
$$


Setting

$$
\mathcal{D}^{ \pm}=\left\{\left(\lambda, \frac{v}{\|v\|^{2}}\right):(\lambda, v) \in \mathcal{C}_{0}^{ \pm}\right\},
$$

we deduce that $\mathcal{D}^{ \pm}$are connected sets of $(\alpha, \Lambda(\alpha)) \times W^{2, p}\left(\mathbb{R}^{N}\right)$ consisting of, respectively, positive and negative solutions of (1.1) with

$$
\inf P \mathcal{D}^{ \pm}=\alpha, \quad \sup P \mathcal{D}^{ \pm}=\Lambda(\alpha) \quad \text { and } \quad\|u\| \geqslant T \quad \text { for all }(\lambda, u) \in \mathcal{D}^{ \pm} .
$$

Suppose that $\left\{\left(\lambda_{n}, u_{n}\right)\right\} \subset \mathcal{D}^{ \pm}$with $\lambda_{n} \stackrel{n}{\rightarrow} \lambda>\alpha$ and $\max _{x \in \mathbb{R}^{N}}\left|u_{n}\right| \stackrel{n}{\rightarrow} \infty$. Then $\left\|u_{n}\right\| \stackrel{n}{\rightarrow} \infty$ by the Sobolev embedding. Hence $\left(\lambda_{n}, v_{n}\right) \in \mathcal{C}$ with $v_{n}=u_{n} /\left\|u_{n}\right\|^{2}$, and $\left\|v_{n}\right\| \stackrel{n}{\rightarrow} 0$. By Theorem 5.2(iii), $\lambda=\Lambda(\alpha)$. On the other hand, if $\left\{\left(\lambda_{n}, u_{n}\right)\right\} \subset \mathcal{D}^{ \pm}$ with $\lambda_{n} \stackrel{n}{\rightarrow} \Lambda(\alpha)$, by setting $v_{n}=u_{n} /\left\|u_{n}\right\|^{2}$ we know that $\left(\lambda_{n}, v_{n}\right) \in \mathcal{C}$ and then $\left\|v_{n}\right\| \stackrel{n}{\rightarrow} 0$ by Theorem 5.2 , which means that $\left\|u_{n}\right\| \stackrel{n}{\rightarrow} \infty$. Moreover, we claim that $\max _{x \in \mathbb{R}^{N}}\left|u_{n}\right| \stackrel{n}{\rightarrow} \infty$, since otherwise, by passing to a subsequence, we may suppose that there is $C>0$ such that $\max _{x \in \mathbb{R}^{N}}\left|u_{n}\right| \leqslant C$ for all $n \in \mathbb{N}$. But $\left(\lambda_{n}, u_{n}\right)$ is a solution of problem (1.1), so we have

$$
L\left(\lambda_{n}\right) u_{n}+k\left(u_{n}\right) u_{n}=0,
$$

which implies that $u_{n} L\left(\lambda_{n}\right) u_{n} \leqslant 0$. Then by Lemma 3.4, $\left\{u_{n}\right\}$ is bounded in $L^{p}\left(\mathbb{R}^{N}\right)$. Therefore, $\left\{(-\Delta+1) u_{n}\right\}$ is bounded in $L^{p}\left(\mathbb{R}^{N}\right)$ since

$$
(-\Delta+1) u_{n}=(\alpha+1) u_{n}+\lambda_{n} g u_{n}+k\left(u_{n}\right) u_{n}
$$

by (5.10). But $-\Delta+1: X \rightarrow Y$ is an isomorphism (see [22, Theorem 2.14] for example), and this implies that $\left\{u_{n}\right\}$ is bounded in $W^{2, p}\left(\mathbb{R}^{N}\right)$, a contradiction.

\section{Appendix A. On global bifurcation}

Let $X$ and $Y$ be real Banach spaces and let $P: \mathbb{R} \times X \rightarrow \mathbb{R}$ denote the projection $P(\lambda, u)=\lambda$. We use the following notation: $\mathcal{B}(X, Y)$ is the space of bounded linear operators from $X$ into $Y$ with its usual norm,

$$
\Phi_{0}(X, Y)=\{L \in \mathcal{B}(X, Y): L \text { is a Fredholm operator of index zero }\}
$$

and

$$
G L(X, Y)=\{L \in \mathcal{B}(X, Y): L: X \rightarrow Y \text { is an isomorphism }\}
$$

Theorem A.1. Let $L \in C^{1}(J, \mathcal{B}(X, Y))$ where $J$ is an open interval and $L(\lambda) \in$ $\Phi_{0}(X, Y)$ for all $\lambda \in J$. Suppose that $\lambda_{0} \in J$ is such that $\operatorname{dim} \operatorname{ker} L\left(\lambda_{0}\right)$ is odd and that

$$
\left[L^{\prime}\left(\lambda_{0}\right) \operatorname{ker} L\left(\lambda_{0}\right)\right] \oplus \operatorname{rge} L\left(\lambda_{0}\right)=Y .
$$

Let $K \in C(X, Y)$ be such that $K: X \rightarrow Y$ is compact and

$$
\lim _{\|u\|_{X} \rightarrow 0} \frac{\|K(u)\|_{Y}}{\|u\|_{X}}=0 .
$$

Let

$$
\widetilde{\mathcal{Z}}=\mathcal{Z} \cup\left\{\left(\lambda_{0}, 0\right)\right\} \quad \text { where } \mathcal{Z}=\{(\lambda, u) \in J \times X: u \neq 0 \text { and } L(\lambda) u+K(u)=0\}
$$

be considered with the metric inherited from $\mathbb{R} \times X$, and let $\mathcal{C}$ denote the connected component of $\widetilde{\mathcal{Z}}$ containing $\left(\lambda_{0}, 0\right)$. Then $\mathcal{C}$ has at least one of the following 
properties:

(i) $\mathcal{C}$ is an unbounded subset of $\mathbb{R} \times X$;

(ii) $\overline{\mathcal{C}} \cap[J \times\{0\}] \neq\left\{\left(\lambda_{0}, 0\right)\right\}$, where $\overline{\mathcal{C}}$ is the closure of $\mathcal{C}$ in $J \times X$;

(iii) either $\sup P \mathcal{C}=\sup J$ or $\inf P \mathcal{C}=\inf J$.

Remarks A.1. (1) The condition (A.1) implies that there exists $\varepsilon>0$ such that $\left[\lambda_{0}-\varepsilon, \lambda_{0}+\varepsilon\right] \subset J, L(\lambda) \in G L(X, Y)$ for all $\lambda \in\left[\lambda_{0}-\varepsilon, \lambda_{0}+\varepsilon\right] \backslash\left\{\lambda_{0}\right\}$, and

$$
\pi\left(L,\left[\lambda_{0}-\varepsilon, \lambda_{0}+\varepsilon\right]\right)=(-1)^{\operatorname{dim} \operatorname{ker} L\left(\lambda_{0}\right)}
$$

where $\pi\left(L,\left[\lambda_{0}-\varepsilon, \lambda_{0}+\varepsilon\right]\right)$ denotes the parity of the path

$$
L:\left[\lambda_{0}-\varepsilon, \lambda_{0}+\varepsilon\right] \rightarrow \Phi_{0}(X, Y)
$$

See $[\mathbf{7}, \mathbf{8}]$.

(2) For $K \in C(X, Y)$, the condition (A.2) is equivalent to the properties $K(0)=$ 0 and $K: X \rightarrow Y$ is Fréchet differentiable at zero with $K^{\prime}(0)=0$.

Proof of Theorem A.1. Let $F(\lambda, u)=L(\lambda) u+K(u)$. We begin by establishing an appropriate property concerning the properness of $F$.

Let $W$ be a bounded subset of $\mathbb{R} \times X$ such that

$$
\inf J<\inf P W \leqslant \sup P W<\sup J
$$

and let $S$ be a compact subset of $Y$. We claim that $W \cap F^{-1}(S)$ is a compact subset of $\mathbb{R} \times X$.

To see this, consider a sequence $\left\{\left(\lambda_{n}, v_{n}\right)\right\} \subset W \cap F^{-1}(S)$. Then $F\left(\lambda_{n}, v_{n}\right) \in S$ and, by passing to a subsequence, we may suppose that

$$
\begin{aligned}
& F\left(\lambda_{n}, v_{n}\right) \rightarrow y \text { strongly in } Y \text { by the compactness of } S, \\
& K\left(v_{n}\right) \rightarrow z \text { strongly in } Y \text { by the compactness of } K, \\
& \left.\lambda_{n} \rightarrow \lambda \in J \text { by the compactness of [inf } P W, \sup P W\right]
\end{aligned}
$$

Hence

$$
\begin{aligned}
L(\lambda) v_{n} & =\left[L(\lambda)-L\left(\lambda_{n}\right)\right] v_{n}+L\left(\lambda_{n}\right) v_{n} \\
& =\left[L(\lambda)-L\left(\lambda_{n}\right)\right] v_{n}+F\left(\lambda_{n}, v_{n}\right)-K\left(v_{n}\right) \\
& \rightarrow 0+y-z \quad \text { strongly in } Y,
\end{aligned}
$$

since $\left\|L(\lambda)-L\left(\lambda_{n}\right)\right\| \rightarrow 0$. But since $L(\lambda) \in \Phi_{0}(X, Y)$, there exist $T \in \mathcal{B}(Y, X)$ and a compact linear operator $C: X \rightarrow X$ such that $T L(\lambda)=I+C$; see $[\mathbf{5}$, Chapter I, Theorem 3.15] for example. Hence $(I+C) v_{n} \rightarrow T(y-z)$ strongly in $X$. Now, passing to a further subsequence, we may suppose that $C\left(v_{n}\right) \rightarrow u$ strongly in $X$ and hence $v_{n} \rightarrow T(y-z)-u$ strongly in $X$, establishing the compactness of $W \cap F^{-1}(S)$.

Given this kind of properness, we find that the conclusion of the theorem is a simple variant of [18, Theorem 9.1] where a degree for continuous compact perturbations of $C^{1}$-Fredholm maps of index zero is defined; see also [21]. The degree for compact perturbations of $C^{1}$-Fredholm maps of index zero has also been developed by Benevieri and Furi (and communicated in a private communication). In fact, in the above setting, it is not necessary to appeal to the full degree theory for compact perturbations of $C^{1}$-Fredholm maps of index zero that are proper on closed bounded sets. As Professor P. J. Rabier pointed out to us, under our assumptions, the map $L(\lambda)+K: X \rightarrow Y$ can be reduced to a compact perturbation 
of the identity on $X$ that depends continuously on $\lambda \in J$ by the introduction of a parametrix for the path $L \in C\left(J, \Phi_{0}(X, Y)\right)$.

\section{Appendix B. On asymptotic linearity}

Definition. Let $X$ and $Y$ be real Banach spaces. A map $M: X \rightarrow Y$ is asymptotically linear if there exists $L \in \mathcal{B}(X, Y)$ such that

$$
\|M(u)-L(u)\|_{Y} /\|u\|_{X} \rightarrow 0 \quad \text { as }\|u\|_{X} \rightarrow \infty
$$

where $L$ is called the asymptotic derivative of $M$.

ExAmPLE. Let $k \in C(\mathbb{R}, \mathbb{R})$ be such that $\lim _{|t| \rightarrow \infty} k(t)=0$. Then the function $M=k(t) t: \mathbb{R} \rightarrow \mathbb{R}$ is asymptotically linear with asymptotic derivative $L=0$.

However, this property is not always inherited by the Nemytskii operator associated with $M$.

Lemma B.1. Let $k \in C(\mathbb{R}, \mathbb{R})$ be such that $k(0) \neq 0$ and $\lim _{|t| \rightarrow \infty} k(t)=0$. For some $p \in[1, \infty)$, let $X=W^{2, p}\left(\mathbb{R}^{N}\right)$ and $Y=L^{p}\left(\mathbb{R}^{N}\right)$. For $u \in X, k(u) u \in Y$ and the mapping $M: X \rightarrow Y$ defined by $M(u)=k(u) u$ for $u \in X$ is continuous and bounded. However, $M: X \rightarrow Y$ is not asymptotically linear.

Proof. Let $\theta=\max _{t \in \mathbb{R}}|k(t)|$. Then $|M(t)| \leqslant \theta|t|$ for all $t \in \mathbb{R}$ and so by the fundamental result concerning Nemytskii operators (see [25], for example), $M$ maps $Y$ continuously and boundedly into $Y$. Hence, $M: X \rightarrow Y$ is continuous and bounded, since $X$ is continuously embedded in $Y$.

If $M: X \rightarrow Y$ is asymptotically linear, there exists $L \in \mathcal{B}(X, Y)$ such that $\|M(u)-L u\|_{Y} /\|u\|_{X} \rightarrow 0$ as $\|u\|_{X} \rightarrow \infty$. First we show that $L \equiv 0$ and then we show that this leads to a contradiction.

Consider any $\varphi \in C_{0}^{\infty}\left(\mathbb{R}^{N}\right)$ such that $\varphi \not \equiv 0$. Then $\|t \varphi\|_{X} \rightarrow \infty$ and so

$$
\|M(t \varphi)-L(t \varphi)\|_{Y} /\|t \varphi\|_{X} \rightarrow 0 \quad \text { as }|t| \rightarrow \infty .
$$

But

$$
\|M(t \varphi)-L(t \varphi)\|_{Y} /\|t \varphi\|_{X}=\|k(t \varphi) \varphi-L \varphi\|_{Y} /\|\varphi\|_{X}
$$

and $\|k(t \varphi) \varphi\|_{Y} \rightarrow 0$ as $|t| \rightarrow \infty$ by dominated convergence. Hence $L \varphi=0$ for all $\varphi \in C_{0}^{\infty}\left(\mathbb{R}^{N}\right)$ and so $L \equiv 0$. Therefore

$$
\lim _{\|u\|_{X} \rightarrow \infty}\|M(u)\|_{Y} /\|u\|_{X}=0 .
$$

Since $k(0) \neq 0$, there exist $\delta>0$ and $T>0$ such that $|k(t)| \geqslant \delta$ for all $|t| \leqslant T$. Choose some $u \in X \backslash\{0\}$ such that $u(x) \in[-T, T]$ for all $x \in \mathbb{R}^{N}$. For $\tau>0$, set $u_{\tau}(x)=u(\tau x)$ for $x \in \mathbb{R}^{N}$. Then $u_{\tau} \in X$ with

$$
\left\|u_{\tau}\right\|_{Y}^{p}=\tau^{-N}\|u\|_{Y}^{p}
$$

and

$$
\left\|u_{\tau}\right\|_{X}^{p}=\tau^{-N}\|u\|_{Y}^{p}+\tau^{p-N} \sum_{i=1}^{N}\left\|\partial_{i} u\right\|_{Y}^{p}+\tau^{2 p-N} \sum_{i, j=1}^{N}\left\|\partial_{i} \partial_{j} u\right\|_{Y}^{p}
$$


In particular, $\left\|u_{\tau}\right\|_{X}^{p} \rightarrow \infty$ as $\tau \rightarrow 0$ since $\|u\|_{Y} \neq 0$. However,

$$
\begin{aligned}
\frac{\left\|M\left(u_{\tau}\right)\right\|_{Y}^{p}}{\left\|u_{\tau}\right\|_{X}^{p}} & =\frac{1}{\left\|u_{\tau}\right\|_{X}^{p}} \int_{\mathbb{R}^{N}}\left|k(u(\tau x)) u_{\tau}(x)\right|^{p} d x \\
& \geqslant \delta^{p} \frac{\left\|u_{\tau}\right\|_{Y}^{p}}{\left\|u_{\tau}\right\|_{X}^{p}}=\delta^{p} \frac{\|u\|_{Y}^{p}}{\tau^{N}\left\|u_{\tau}\right\|_{X}^{p}}
\end{aligned}
$$

where $\tau^{N}\left\|u_{\tau}\right\|_{X}^{p} \rightarrow\|u\|_{Y}^{p}$ as $\tau \rightarrow 0$. Hence $\liminf _{\tau \rightarrow 0}\left\|M\left(u_{\tau}\right)\right\|^{p} /\left\|u_{\tau}\right\|_{X}^{p} \geqslant \delta^{p}$, contradicting (B.1). Thus $M$ cannot be asymptotically linear.

Lemma B.2. Under the hypotheses of Lemma B.1 with $p>\min \left\{1, \frac{1}{2} N\right\}$, let $\psi \in L^{1}\left(\mathbb{R}^{N}\right) \cap L^{\infty}\left(\mathbb{R}^{N}\right)$. The mapping $Q: X \rightarrow Y$ defined by $Q(u)=\psi k(u) u$ for $u \in X$ is continuous, bounded, compact and asymptotically linear with asymptotic derivative equal to zero.

Proof. Since $M=k(u) u: X \rightarrow Y$ is continuous and bounded by Lemma B.1 and $\psi \in L^{\infty}\left(\mathbb{R}^{N}\right)$, it follows that $Q: X \rightarrow Y$ is continuous and bounded. Choose some $t \in(1, \infty)$ such that $X$ is continuously embedded in $L^{t p}\left(\mathbb{R}^{N}\right)$ and let $s=$ $t /(t-1)$. For any $R>0$ and any $u \in X$,

$$
\begin{aligned}
\int_{|x| \geqslant R}|\psi k(u) u|^{p} d x & \leqslant|k|_{\infty}^{p}\left\{\int_{|x| \geqslant R}|\psi|^{s p} d x\right\}^{1 / s}\left\{\int_{|x| \geqslant R}|u|^{t p} d x\right\}^{1 / t} \\
& \leqslant|k|_{\infty}^{p}\left\{\int_{|x| \geqslant R}|\psi|^{s p} d x\right\}^{1 / s}|u|_{t p}^{p} \\
& \leqslant C(t)|k|_{\infty}^{p}\|u\|_{X}^{p}\left\{\int_{|x| \geqslant R}|\psi|^{s p} d x\right\}^{1 / s} .
\end{aligned}
$$

Given any $\varepsilon>0$, we can choose $R>0$ such that $\int_{|x| \geqslant R}|\psi|^{s p} d x<\varepsilon$. Let $B_{R}=\left\{x \in \mathbb{R}^{N}:|x|<R\right\}$. Since $W^{2, p}\left(B_{R}\right)$ is compactly embedded in $C\left(B_{R}\right)$ because $p>\frac{1}{2} N$, one easily deduces the compactness of $Q: X \rightarrow Y$.

Given any $\varepsilon>0$, there exists $S_{\varepsilon}>0$ such that $0 \leqslant k(s)<\varepsilon$ for all $|s| \geqslant S_{\varepsilon}$ by (1.6). For $u \in X \backslash\{0\}$, let

$$
D(u, \varepsilon)=\left\{x \in R^{N}:|u(x)| \geqslant S_{\varepsilon}\right\} .
$$

Then

$$
\begin{aligned}
|\psi k(u) u|_{p}^{p} & \leqslant|\psi|_{\infty}^{p} \varepsilon^{p} \int_{D(u, \varepsilon)}|u|^{p} d x+|k|_{\infty}^{p} S_{\varepsilon}^{p} \int_{\mathbb{R}^{N} \backslash D(u, \varepsilon)}|\psi|^{p} d x \\
& \leqslant|\psi|_{\infty}^{p} \varepsilon^{p}\|u\|_{X}^{p}+|k|_{\infty}^{p} S_{\varepsilon}^{p}|\psi|_{\infty}^{p-1}|\psi|_{1} .
\end{aligned}
$$

Hence

$$
\limsup _{\|u\|_{X} \rightarrow \infty} \frac{|\psi k(u) u|_{p}}{\|u\|_{X}} \leqslant|\psi|_{\infty} \varepsilon \quad \text { for any } \varepsilon>0,
$$

which shows that $Q: X \rightarrow Y$ is asymptotically linear with asymptotic derivative zero. 
LEMma B.3. In addition to the hypotheses of Lemma B.2 suppose that $k \in$ $C^{1}(\mathbb{R}, \mathbb{R})$ and that $|\psi|_{\infty} \neq 0$. Set

$$
K(u)= \begin{cases}\psi k\left(u /\|u\|_{X}^{2}\right) u & \text { if } u \in X \backslash\{0\}, \\ 0 & \text { if } u=0 .\end{cases}
$$

Then $K: X \rightarrow Y$ is Fréchet differentiable at $u$ for all $u \in X$ and we have $K \in C^{1}(X \backslash\{0\}, Y)$. However, $K^{\prime}(0)=0$ but $\limsup _{\|u\|_{X} \rightarrow 0}\left\|K^{\prime}(u)\right\|>0$, so $K \notin C^{1}(X, Y)$.

Proof. First we observe that

$$
K(u)= \begin{cases}\|u\|_{X}^{2} Q\left(u /\|u\|_{X}^{2}\right) & \text { if } u \in X \backslash\{0\}, \\ 0 & \text { if } u=0 .\end{cases}
$$

Using [19, Lemma 5.3(i)], we deduce that $Q \in C^{1}(X, Y)$. Furthermore, using the standard properties of substitution operators, we find that

$$
|\cdot|_{p} \in C^{1}\left(L^{p}\left(\mathbb{R}^{N}\right) \backslash\{0\}, \mathbb{R}\right) ;
$$

see [27, Chapter 1] for example. But if $u \in X$ and either $\partial_{\alpha} u \equiv 0$ or $\partial_{\alpha} \partial_{\beta} u \equiv 0$ for some $\alpha, \beta \in\{1,2, \ldots, N\}$, then $u \equiv 0$. Hence $\|\cdot\|_{X}=|\cdot|_{2, p} \in C^{1}\left(W^{2, p}\left(\mathbb{R}^{N}\right) \backslash\{0\}, \mathbb{R}\right)$ and it follows by composition that $K \in C^{1}(X \backslash\{0\}, Y)$.

For the differentiability of $K$ at $u=0$, we observe that

$$
\begin{aligned}
\lim _{\|u\|_{X} \rightarrow 0} \frac{\|K(u)\|_{Y}}{\|u\|_{X}} & =\lim _{\|u\|_{X} \rightarrow 0}\|u\|_{X}\left\|Q\left(\frac{u}{\|u\|_{X}^{2}}\right)\right\|_{Y} \\
& =\lim _{\|v\|_{X} \rightarrow \infty}\|v\|_{X}^{-1}\|Q(v)\|_{Y}=0
\end{aligned}
$$

by Lemma B.2 with $v=u /\|u\|_{X}^{2}$. Hence $K$ is Fréchet differentiable at $u=0$ with $K^{\prime}(0)=0$.

Let $a, b \in \mathbb{R}^{N}$ with $|a-b|>2$ and let $B_{a}$ and $B_{b}$ denote the open balls of unit radius about these points. Consider two functions $u, v \in C_{0}^{\infty}\left(\mathbb{R}^{N}\right) \backslash\{0\}$ with $\operatorname{supp} u \subset B_{a}$ and $\operatorname{supp} v \subset B_{b}$. Now

$$
\lim _{t \rightarrow 0} \frac{K(u+t v)-K(u)}{t}=K^{\prime}(u) v
$$

and

$$
\begin{aligned}
\left\|\frac{K(u+t v)-K(u)}{t}\right\|_{Y}^{p}= & \int_{B_{a}}\left|\frac{K(u+t v)-K(u)}{t}\right|^{p} d x \\
& +\int_{B_{b}}\left|\frac{K(u+t v)-K(u)}{t}\right|^{p} d x \\
\geqslant & \int_{B_{b}}\left|\frac{K(u+t v)}{t}\right|^{p} d x \\
= & \int_{B_{b}} \frac{\left|\psi k\left(t v /\|u+t v\|_{X}^{2}\right) t v\right|^{p}}{|t|^{p}} d x \\
= & \int_{B_{b}}\left|\psi k\left(\frac{t v}{\|u+t v\|_{X}^{2}}\right) v\right|^{p} d x .
\end{aligned}
$$


Since $\|u\|_{X} \neq 0$, it follows by dominated convergence that

$$
\lim _{t \rightarrow 0} \int_{B_{b}}\left|\psi k\left(\frac{t v}{\|u+t v\|_{X}^{2}}\right) v\right|^{p} d x=|k(0)|^{p} \int_{B_{b}}|\psi v|^{p} d x,
$$

whereas

$$
\left\|K^{\prime}(u) v\right\|_{Y}^{p}=\lim _{t \rightarrow 0}\left\|\frac{K(u+t v)-K(u)}{t}\right\|_{Y}^{p}
$$

and so

$$
\left\|K^{\prime}(u) v\right\|_{Y}^{p} \geqslant|k(0)|^{p} \int_{B_{b}}|\psi v|^{p} d x
$$

Hence

$$
\left\|K^{\prime}(u)\right\|_{B(X, Y)}^{p} \geqslant|k(0)|^{p} \int_{B_{b}}|\psi v|^{p} d x /\|v\|_{X}^{p} .
$$

Since $|\psi|_{\infty} \neq 0$, we can choose $b \in \mathbb{R}^{N}$ such that

$$
\int_{B_{b}}|\psi|^{2 p} d x>0
$$

and this implies that we can choose $v_{\psi} \in C_{0}^{\infty}\left(\mathbb{R}^{N}\right)$ such that $\operatorname{supp} v_{\psi} \subset B_{b}$ and

$$
\int_{B_{b}}\left|\psi v_{\psi}\right|^{p} d x \geqslant \frac{1}{2} \int_{B_{b}}|\psi|^{2 p} d x>0
$$

Thus we see that, for any $u \in C_{0}^{\infty}\left(\mathbb{R}^{N}\right) \backslash\{0\}$ such that $\operatorname{supp} u \subset B_{a}$,

$$
\left\|K^{\prime}(u)\right\|_{B(X, Y)} \geqslant L \quad \text { where } L=|k(0)|\left\{\frac{1}{2} \int_{B_{b}}|\psi|^{2 p} d x\right\}^{1 / p} /\left\|v_{\psi}\right\|_{X} .
$$

Considering a sequence $\left\{u_{n}\right\} \subset C_{0}^{\infty}\left(\mathbb{R}^{N}\right) \backslash\{0\}$ which is such that supp $u_{n} \subset B_{a}$ and $\left\|u_{n}\right\|_{X} \rightarrow 0$, we see that $\lim \sup _{\|u\|_{X} \rightarrow 0}\left\|K^{\prime}(u)\right\|_{B(X, Y)} \geqslant L>0$, completing the proof.

\section{Appendix C. Some topological lemmas}

Lemma C.1. The set $V$ defined in (5.8) is compact.

Proof. Let $\left\{\left(\lambda_{n}, v_{n}\right)\right\} \subset V$, for $n=1,2,3, \ldots$, be an infinite sequence. By the proof of Theorem 5.2(ii), $\mathcal{Q}$ is bounded in $\mathbb{R} \times X$. So, passing to a subsequence, we may suppose that either $v_{n}>0$ for all $n$ or $v_{n}<0$ for all $n$ and

$$
\begin{aligned}
& \lambda_{n} \stackrel{n}{\rightarrow} \lambda \in[\bar{\mu}, \Lambda(\alpha)], \\
& v_{n} \stackrel{n}{\rightarrow} v \text { weakly in } X \text { with } v \geqslant 0 \text { or } v \leqslant 0, \\
& \left\|v_{n}\right\| \stackrel{n}{\rightarrow} \ell \geqslant 0 .
\end{aligned}
$$

To prove the lemma, it is enough to show that

$$
v_{n} \stackrel{n}{\rightarrow} v \text { strongly in } X \text { with }(\lambda, v) \in V .
$$

By $\left(\lambda_{n}, v_{n}\right) \in V \subset \mathcal{Z},(5.3)$ holds with $\psi_{n}(x) \equiv 1$, that is,

$$
L\left(\lambda_{n}\right) v_{n}+k\left(\frac{v_{n}}{\left\|v_{n}\right\|^{2}}\right) v_{n}=0
$$


Then, as in the proof of Theorem 5.2(i), we know that $v_{n}$ satisfies (3.14). We now prove the lemma by considering two cases: $\ell=0$ and $\ell>0$.

Case $(\mathrm{A}): \ell=0$. Since $\ell=0, v_{n} \stackrel{n}{\rightarrow} 0$ strongly in $X$. As in the proof of Theorem 5.2(i), Lemma 3.6 can be used to show that $\lambda=\Lambda(\alpha)$, and we have the required result: $(\Lambda(\alpha), 0) \in V$.

Case (B): $\ell>0$. In this case, we can repeat the proof of (5.4), just replacing $\psi_{n}(x)$ by 1 , to show that $v_{n} \stackrel{n}{\rightarrow} v$ strongly in $X$ and that (5.7) holds with $v^{2}>0$ in $\mathbb{R}^{N}$. Thus $(\lambda, v) \in V$.

The following lemma was proved in [26, Chapter I, $\S 9.3]$.

Lemma C.2 (Whyburn). Suppose that $V_{1}$ and $V_{2}$ are closed subsets of a compact metric space $V$ such that there is no connected component of $V$ that intersects both $V_{1}$ and $V_{2}$. Then there exist disjoint compact sets $U_{1}$ and $U_{2}$ such that

$$
V=U_{1} \cup U_{2}, \quad V_{1} \subset U_{1} \quad \text { and } \quad V_{2} \subset U_{2}
$$

The next lemma is Proposition 5.1 of $[\mathbf{1}]$.

Lemma C.3 [1, Proposition 5.1]. Suppose $A$ and $B$ are closed and not separated in a compact $X$. Then there exists a connected $D$ in $X \backslash\{A \cup B\}$ such that $\bar{D} \cap A \neq \emptyset$, and $\bar{D} \cap B \neq \emptyset$.

\section{References}

1. J. C. Alexander, 'A primer on connectivity', Fixed point theory (ed. A. Dold and B. Eckmann), Lecture Notes in Mathematics 886 (Springer, Berlin, 1980) 455-482.

2. T. BARtsch, A. PAnkov and Z. Q. WANG, 'Nonlinear Schrödinger equations with steep potential well', Commun. Contemp. Math. 3 (2001) 549-569.

3. T. BARTSCH and Z. Q. WANG, 'Multiple positive solutions for a nonlinear Schrödinger equation', Z. angew. Math. Phys. 51 (2000) 366-384.

4. Y. Ding and K. TANAKA, 'Multiplicity of positive solutions of a nonlinear Schrödinger equation', Manuscripta Math. 112 (2003) 109-135.

5. D. E. Edmunds and W. D. Evans, Spectral theory and differential operators (Oxford University Press, 1987).

6. D. G. DE Figueiredo and Y. Ding, 'Solutions of a non-linear Schrödinger equation', Discrete Contin. Dynam. Systems 8 (2002) 563-584.

7. P. M. FitzPAtrick, 'Homotopy, linearization, bifurcation', Nonlinear Anal. 12 (1988) 171184.

8. P. M. Fitzpatrick and J. Pejsachowicz, 'Parity and generalized multiplicity', Trans. Amer. Math. Soc. 326 (1991) 281-305.

9. P. M. Fitzpatrick, J. Pejsachowicz and P. J. Rabier, 'The degree of proper $C^{2}$ Fredholm mappings', J. reine angew. Math. 427 (1992) 1-33.

10. D. Gilbarg and N. S. Trudinger, Elliptic partial differential equations of second order, 2nd edn (Springer, Berlin, 1983).

11. F. A. VAN HeERden, 'Multiple solutions for a Schrödinger type equation with an asymptotically linear term', Nonlinear Anal. 55 (2003) 739-758.

12. F. A. VAN HEERDEN and Z. Q. WANG, 'Schrödinger type equations with asymptotically linear nonlinearities', Differential Integral Equations 16 (2003) 257-280.

13. H. Jeanjean, M. LuCia and C. A. Stuart, 'Branches of solutions to semilinear elliptic equations on $\mathbb{R}^{N}$, Math. Z. 230 (1999) 79-105.

14. L. JEANJEAN and K. TANAKA, 'A positive solution for an asymptotically linear elliptic problem on $\mathbb{R}^{N}$ autonomous at infinity', ESAIM Control Optim. Calc. Var. 7 (2002) 597-614.

15. Z. LiU and Z.-Q. WANG, 'Existence of a positive solution of an elliptic equation on $\mathbb{R}^{N}$ ', Proc. Royal Soc. Edinburgh Sect. A 134 (2004) 191-200.

16. J. Pejsachowicz and P. J. Rabier, 'Degree theory for $C^{1}$ Fredholm mapping of index zero', J. Anal. Math. 76 (1998) 289-319. 
17. P. J. RABIER, 'Invariance of the $\Phi_{0}$-spectrum and Sobolev regularity for second order elliptic problems on $\mathbb{R}^{N}$, Applicable mathematics in the golden age (ed. J. C. Misra; Narosa, New Delhi, 2003) 1-31.

18. P. J. RABier and M. F. SAlter, 'A degree theory for compact perturbations of proper $C^{1}$-Fredholm mappings of index zero', Abstr. Appl. Anal. 2005 (2005) 707-731.

19. P. J. RABier and C. A. Stuart, 'Application of elliptic regularity to bifurcation in stationary nonlinear Schrödinger equations', Nonlinear Anal. 52 (2003) 869-890.

20. P. H. Rabinowitz, 'On bifurcation from infinity', J. Differential Equations 14 (1973) 462-475.

21. M. F. SAlter, 'Degree theory for compact perturbations of Fredholm maps of index zero', $\mathrm{PhD}$ Thesis, University of Pittsburgh, 2002.

22. C. A. Stuart, 'An introduction to elliptic equations on $\mathbb{R}^{N}$, Nonlinear functional analysis and applications to differential equations (ed. A. Ambrosetti, K.-C. Chang and I. Ekeland; World Scientific, Singapore, 1998).

23. C. A. Stuart and H.-S. Zhou, 'Positive eigenfunctions of a Schrödinger operator', J. London Math. Soc. (2) 72 (2005) 429-441.

24. J. F. Toland, 'Asymptotic nonlinearity and nonlinear eigenvalue problems', Quart. J. Math. Oxford 24 (1973) 241-250.

25. M. M. VAinberg, Variational methods for the study of nonlinear operators (Holden-Day, San Francisco, 1964).

26. G. T. Whyburn, Topological analysis (Princeton University Press, 1958).

27. M. Willem, Minimax theorems (Birkhäuser, Boston, 1996).

C. A. Stuart

IACS-FSB

Section de Mathématiques

École Polytechnique Fédérale de Lausanne

CH-1015 Lausanne

Switzerland

charles.stuart@epfl.ch
Huan-Song Zhou

Wuhan Institute of Physics

and Mathematics

Chinese Academy of Sciences

P.O. Box 71010

Wuhan 430071

P. R. China

hszhou@wipm.ac.cn 